\title{
INDICADORES TECNOLÓGICOS PARA LA SELECCIÓN DE PLATAFORMAS MOOC
}

\section{TECHNOLOGICAL INDICATORS FOR THE SELECTION OF MOOC PLATFORMS}

\author{
Susan Yulieth Huertas Lòpez ${ }^{1}$ \\ Fredy Yesid Mesa Jiménez ${ }^{2}$ \\ Universidad Pedagógica y \\ Tecnológica de Colombia
}

\section{RESUMEN}

La utilidad de las plataformas de Cursos Abiertos Masivos en Línea o MOOC (Massive Open Online Courses) permite desarrollar experiencias formativas personalizadas, con la ventaja de que estos cursos son ofrecidos a través de entornos virtuales, los cuales pueden ser accedidos a cualquier hora y desde cualquier parte del mundo, por medio del internet. Dada la alta oferta en educación electrónica, ¿Cómo se puede seleccionar un curso que satisfaga los criterios de calidad y confiabilidad? Este estudio busca analizar los aspectos tecnológicos que intervienen en la calidad de un curso virtual. $1 \quad$ Universidad Pedagógica y Tecnológica de Colombia. susanyulieth@gmail.com de Colombia. fredy.mesa@uptc.edu.co
Para tal fin se realiza una revisión documental, encuestas, observaciones y exploraciones para establecer algunos indicadores que permitan la valoración de aspectos tecnológicos en plataformas MOOC, permitiendo establecer indicadores en tres tipologías: 1) tecnología de equipo, 2) tecnología de operación y 3) tecnología de producto, a través de los cuales se puede realizar una valoración cuantitativa y cualitativa de manera secuencial dentro del desarrollo de un curso, entonces si se quiere realizar un MOOC se debe establecer los componentes tecnológicos requeridos de acuerdo a la necesidad. 


\section{PALABRAS CLAVE}

Indicadores; informática; tecnología; educación abierta, criterio de selección.

\section{ABSTRACT}

The usefulness of the Massive Open Online Courses platforms allows to develop personalized training experiences, with the advantage that these courses are offered through virtual environments, which can be accessed at any time and from anywhere in the world, through the internet. Given the high offer in electronic education, how can you select a course that meets the criteria of quality and reliability? This study seeks to analyze the technological aspects that intervene in the quality of a virtual course. For this purpose, a documentary review, evaluations, observations and explorations are carried out to establish some indicators that affect the assessment of technological aspects in MOOC platforms, that establish indicators in three typologies: 1) equipment technology, 2) operation technology and 3) technology of product, through which a quantitative and qualitative assessment can be carried out sequentially within the development of a course, then if you want to perform a MOOC you must establish the required technological components according to the need.

\section{KEYWORDS}

Indicator; computing; technology; open education, selection criteria.

\section{INTRODUCCIÓN}

Los Cursos Online Masivos en Abierto (COMA) o en inglés Massive Open Online Course (MOOC), son realizados de acuerdo a la necesidad de los usuarios, en algunas ocasiones para complementar o profundizar conocimientos en un área determinada o como un medio de actualización dirigido a empleados de empresas, generalmente con el respaldo de Universidades, Institutos o empresas.

Para las Universidades los MOOC son una oportunidad de internacionalizarse y extender su oferta educativa (en especial posgrados), homologar conocimientos y obtener recursos económicos adicionales. El estudio de la influencia de las plataformas MOOC en la educación, es un proyecto relevante para las instituciones de educación en todos sus niveles (Eloy, 2015, Kinash, 2013).

Los MOOC, son una solución a algunas limitaciones asociadas al modelo tradicional de clase magistral; por ende, desde la digitalización de contenidos en una plataforma se puede llegar a obtener mayor volumen de estudiantes sin restricciones de tiempo, coste o espacio, como lo expresa Bailador (2014), convirtiéndose en una herramienta útil, en el contexto de una educación abierta basada en las TIC.

Dado que las plataformas MOOC cuentan con la facilidad de implementarse con contenidos de interés y un marco de temas por seleccionar (Alemán de la Garza, 2015), además, permite estudiar desde la comodidad de la casa ahorrando costos, tiempo, espacio y recursos, entre otros.

Dentro de las desventajas, es notorio que la didáctica desarrollada en los MOOC, se basa en presentar contenidos de forma expositiva y no exige mucha iniciativa por parte del estudiante (Meiss, 2017), también, algunos MOOC no aprovechan al máximo las potencialidades de las TIC.

Por otro lado, la deserción en la educación virtual es alta, ya que solo un $5 \%$ de las personas que cursan un MOOC lo terminan (Ruiz, 2015), lo que conlleva a analizar aspectos de la calidad de estos cursos, visto desde los usuarios y sus convicciones por este tipo educación. 
El tiempo es una de las causas de deserción (Ruiz, 2015), puesto que, si no se realiza el curso en los tiempos establecidos, se atrasa en los contenidos y puede que hasta se cierre el curso y no se pueda continuar.

La presente investigación propone un marco de trabajo con base en el análisis, comparación y evaluación de las plataformas MOOC, desde el punto de vista tecnológico, brindando un criterio necesario para que las universidades que deseen extenderse por este medio y los docentes y estudiantes que deseen experimentar un aprendizaje autónomo y colaborativo mediado por estos entornos virtuales, tengan unos criterios para su selección.

Algunas plataformas asignan sus lineamientos, esquemas de diseño y desarrollo para los MOOC, mientras que otras dan libertad a los creadores de los cursos, por lo cual, no todos los usuarios conocen y aprovechan las potencialidades de estas plataformas

\section{METODOLOGÍA}

Se realizó un análisis documental y una revisión de cada una de las páginas web de las plataformas estudiadas, para realizar su comparación (ver tabla 1). Además, se diseñó y aplicó una encuesta virtual a través del aplicativo www.e-encuesta.com ${ }^{3}$ con preguntas de tipo tecnológico y pedagógico.

\section{METODOLOGÍA PARA LA FORMULACIÓN DE INDICADORES}

Para la selección de plataformas MOOC, se adaptaron las siguientes guías: 1) Guía metodológica para la formulación de indicadores del Departamento Nacional de Planeación de Colombia (DNP, 2009), 2) guía para diseño, construcción e interpretación de indicadores, de autoría del DepartamentoAdministrativo Nacional de Estadística de Colombia (DANE) y 3) guía de indicadores del Ministerio de Tecnologías de la Información y las Comunicaciones de Colombia (MINTIC). De estos documentos se obtuvieron pautas generales, que se tomaron como base para la generación de los indicadores de calidad para MOOC.

Para DNP(2009), un indicador es la presentación cuantitativa, verificable objetivamente, a partir de la cual se registra, procesa y presenta la información necesaria para medir el avance o retroceso en el logro de un determinado objetivo. 3 Se utilizó la inscripción a la versión gratuita.

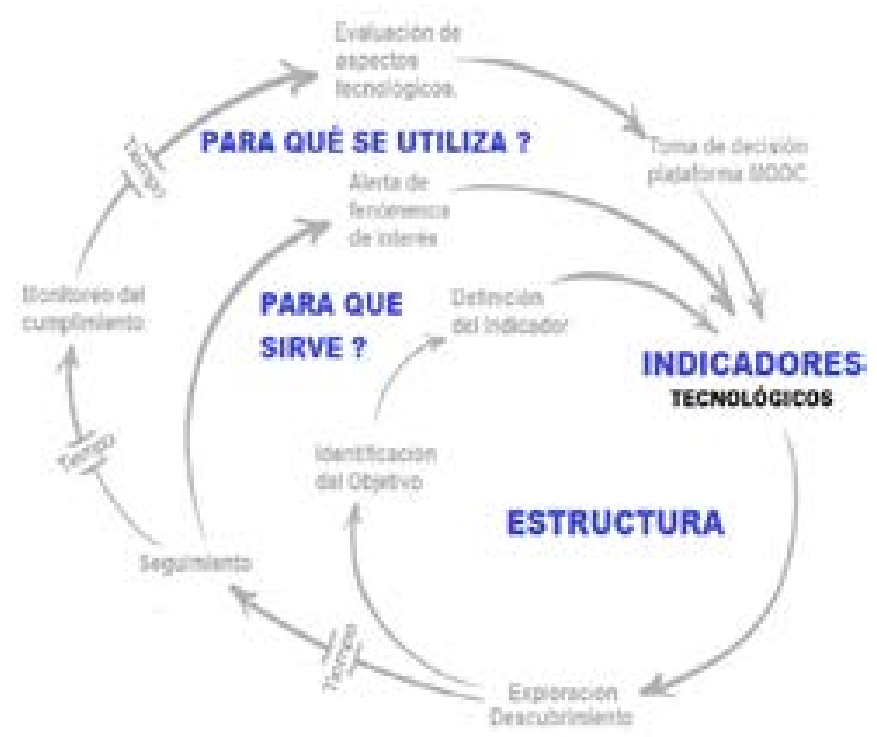

Figura 1: Funcionalidad del indicador de un MOOC, modificado y adaptado de la imagen tomada de https://cabreramc.files.wordpress.com/2015/04/marco_transformaciocc81n_ff.jpg 
La composición del indicador se centra en el objeto de toma de decisión presente en un fenómeno dentro de un contexto, claramente los indicadores son dados por el flujo de datos que permite el muestreo de medición cuantitativamente o cualitativamente, como se muestra en la figura 1 , dentro del proceso de desarrollo del indicador está dado por el seguimiento continuo en espacios de tiempo establecidos como unidad de medida en la variación del cumplimiento del indicador (Astros, 2015).

\section{AUTOPERCEPCIÓN DE USUARIOS DE PLATAFORMAS SOBRE ASPECTOS TECNOLÓGICOS DE MOOC}

Se aplicó una encuesta a una muestra de 25 estudiantes que han cursado MOOC, de las maestrías en Ambientes Educativos Mediados por TIC (14 estudiantes) y Tecnología en Informática (11 estudiantes) de la Universidad Pedagógica y Tecnológica de Colombia (UPTC). El muestreo fue no probabilístico, por conveniencia, debido a la facilidad de acceso que se tuvo a grupos de estudiantes de posgrado.

El $45 \%$ de los encuestados son de formación en Ingeniería de Sistemas o Licenciados en Informática y Tecnología (estudian para ser profesores) y el porcentaje restante son de otras carreras ofrecidas por la UPTC.

El $43 \%$ de los encuestados corresponde a mujeres y el $57 \%$ a hombres. El $43 \%$ oscilan entre edades de los 19 a 25 años, un $48 \%$ entre 26 a 40 años y el $10 \%$ restante son personas mayores de 40 años.

Por otro lado, se envió un cuestionario al servicio de ayuda o al correo electrónico de cada una de las 25 plataformas descritas (ver tabla 1), de las cuales respondieron al mensaje FutureLearn ${ }^{4}$, Academic Earth ${ }^{5}$, Chehena ${ }^{6}$, Coursera ${ }^{7}$ y edX ${ }^{8}$.

\section{RESULTADOS}

\section{Comparación de algunas plataformas MOOC}

Para (Hernández, 2015) y (Garrido, 2003), las plataformas MOOC son la transformación de la Educación Superior, por medio de un diseño modular (descentralizado, basado en necesidades reales, dinámicas y adaptables) y desechando un diseño interdependiente (rígido, estandarizado e inflexible). Lo anterior, lo muestra como un medio para flexibilizar el aprendizaje en cualquier entorno educativo.

A continuación, se presenta un análisis de las plataformas MOOC más usadas y conocidas de acuerdo con Escribano (2014), Lorente (2017) y Olmo (2017) (Ver tabla 1). Análisis de las plataformas MOOC más reconocidas). La descripción fue realizada con base en información obtenida de los sitios web.

En la comparación realizada de las plataformas, algunas difieren en sus costos en mensualidad o por pago anual, para incluir servicios adicionales (de los cuales no se encontró mayor información al respecto) a los ofrecidos en la versión gratuita.

4 Del correo enviado se obtuvo la siguiente respuesta: "Recibimos muchas solicitudes de investigación de académicos que solicitan ayuda con su investigación. No podemos cumplir con todo esto, así que me temo que esto no es algo con lo que podamos ayudar, ¡lo siento!"

5 Mediante correo se notifica "Cualquier información que requieras con respecto a nuestros MOOC debes concertar una cita para poder brindarte la información que requieres".

$6 \quad$ Solicitud enviada por el servicio de ayuda, se da como respuesta "Hola, hemos recibido tu solicitud (65308) y nuestro equipo de Customer Happiness te responderá lo más pronto posible. Tenemos muchos mensajes en cola y te recomendamos verificar si tu consulta se encuentra aquí"

7 "Lamento los inconvenientes, pero lastimosamente no podemos responder a las preguntas de la encuesta debido a que solo tenemos permitido aclarar preguntas acerca de los cursos que el alumno pueda tener, así como resolver problemas técnicos que se puedan presentar. De igual manera le puedo pedir amablemente que ingrese a nuestro Centro de Ayuda de Coursera ya que allí podrá encontrar muchas de las preguntas que nos solicitó."

8 Respuesta de la plataforma "Le contactaremos pronto. Responderemos normalmente en un día laborable. Mientras tanto, mire en nuestro Centro de ayuda. Puede que encuentre su respuesta ahi". 
También se evidenció, que parte de las plataformas exigen a los creadores de MOOC que la información que se presente en los cursos sea clara, precisa, concisa y actual, pues de ello depende la calidad de los cursos.

Las políticas de privacidad y los términos de servicio presentados en las plataformas, coinciden en aplicar principios éticos manejados al interior de la comunidad virtual, los cuales son centrados en la responsabilidad y respeto académico, así también, para otras la restricción del condicionamiento de edad, ya que los usuarios deben contar con más de 13 años y con permiso de un adulto responsable. De otra forma, si se incurre en aspectos legales, los datos del usuario son compartidos ante las autoridades.

En cuanto se trata de la adquisición de un certificado respaldado por una de las entidades socias, normalmente se requiere de realizar un pago por este documento, generalmente a petición del usuario. Algunas plataformas verifican el conocimiento adquirido, confrontando al usuario a través de videoconferencia e inclusive validación biométrica, para el reconocimiento del certificado. En caso de que un usuario pague desde el inicio del curso y no esté satisfecho, algunas plataformas ofrecen la devolución del dinero (Udacity, edX, FutureLearn, Udemy, Crehena y Canvas Network).
Algunas universidades Colombianas que participan en plataformas de MOOC, son: 1) en Miríada X, la Universidad Cooperativa de Colombia, Uniminuto, Universidad del Norte, Universidad del Bosque, Universidad Autónoma de Occidente, Universidad ICESI, Universidad del Rosario, Universidad CES, Universidad Externado de Colombia, Universidad EAFIT, Universidad de Ibagué y el CEPEI (Centro de Pensamiento estratégico Internacional); 2) en Coursera, participa la Universidad de los Andes y en 3) edX, la Universidad del Rosario. Tabla 1: 


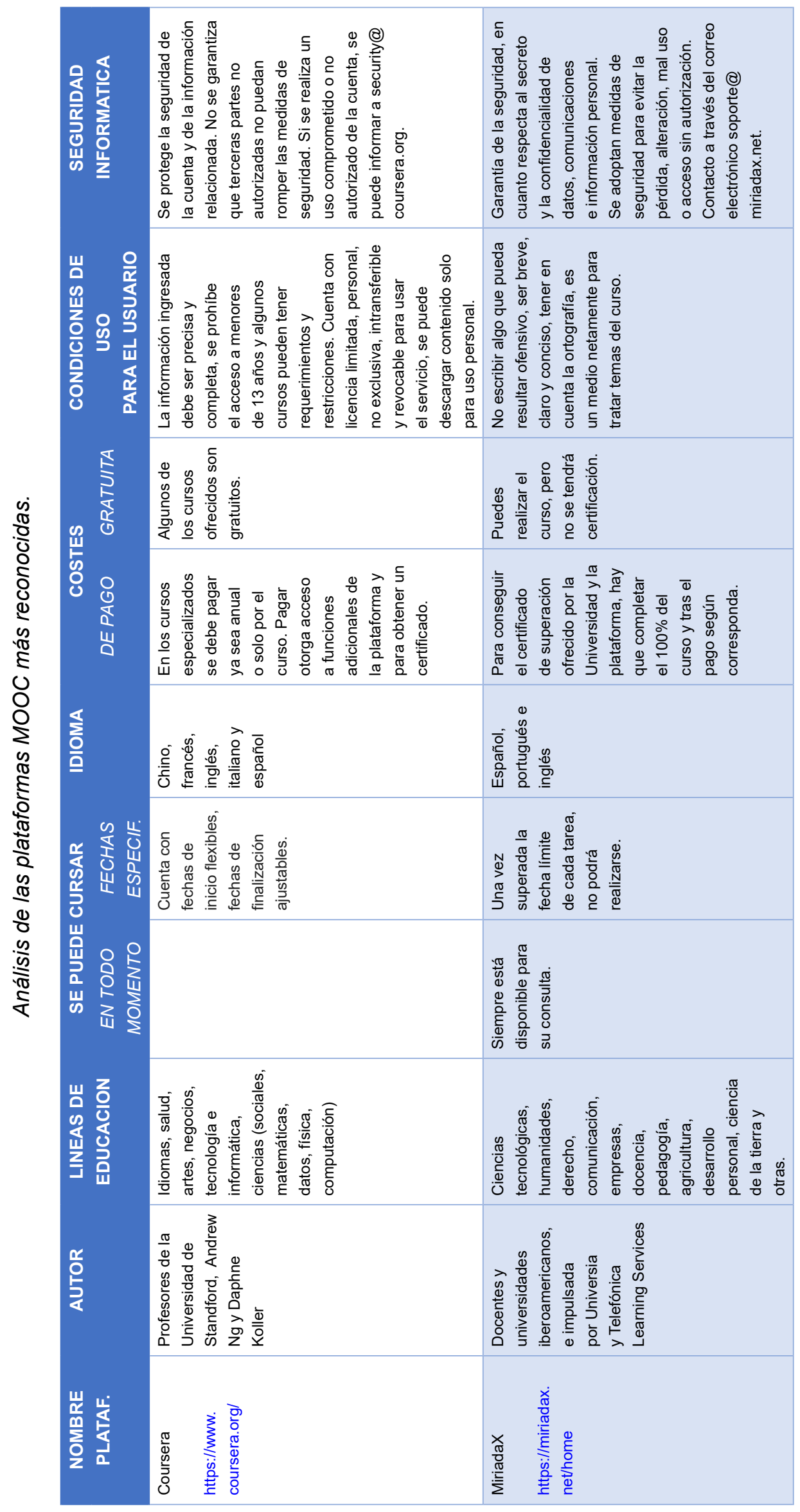




\begin{tabular}{|c|c|c|c|}
\hline 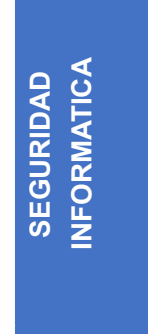 & 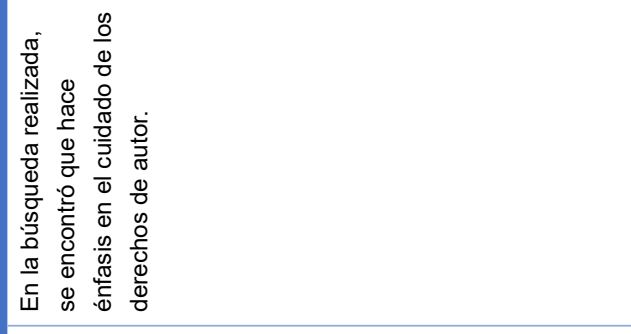 & 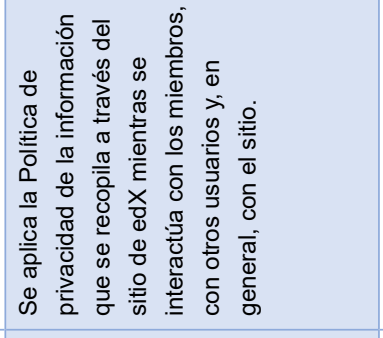 & 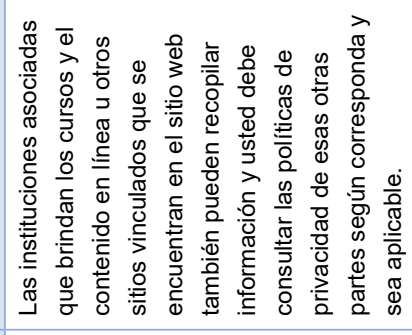 \\
\hline 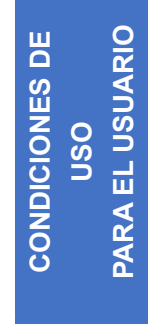 & 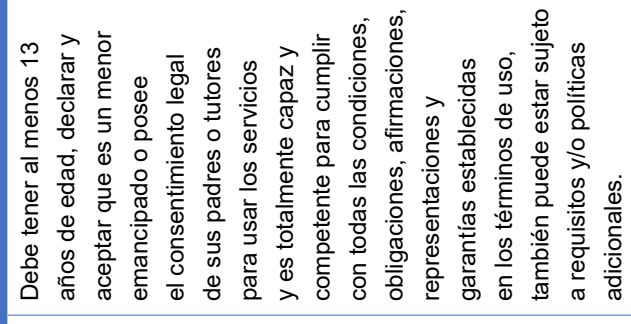 & 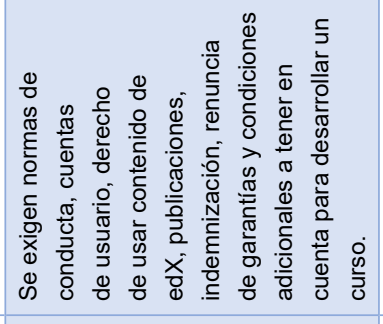 & 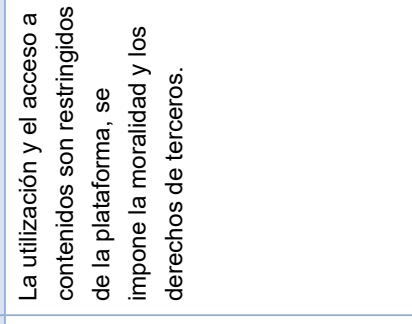 \\
\hline 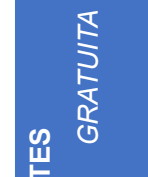 & 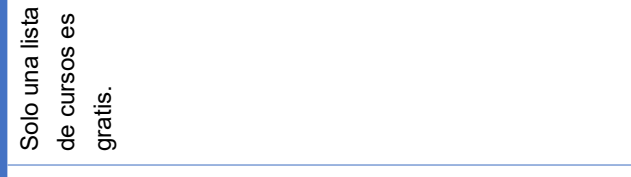 & 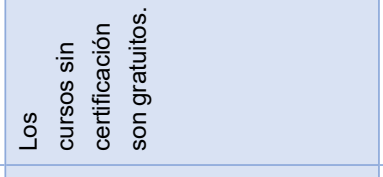 & 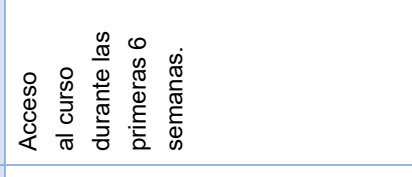 \\
\hline 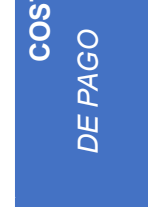 & 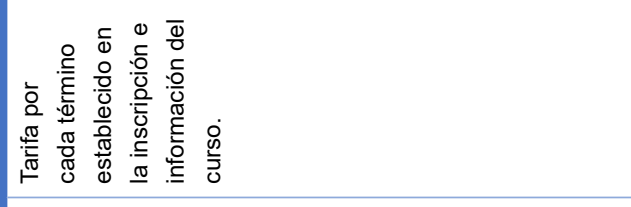 & 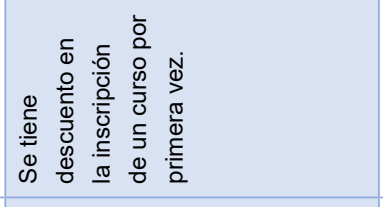 & 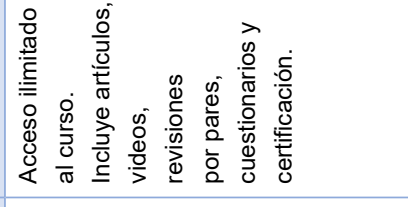 \\
\hline & 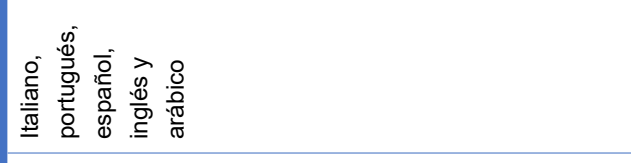 & 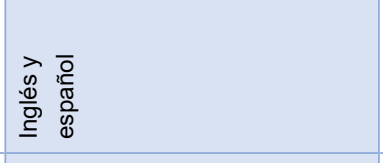 & $\begin{array}{l}\frac{\mathscr{w}}{\underline{\underline{D}}} \\
\underline{\underline{\Xi}}\end{array}$ \\
\hline 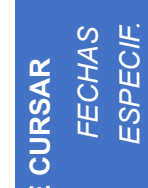 & 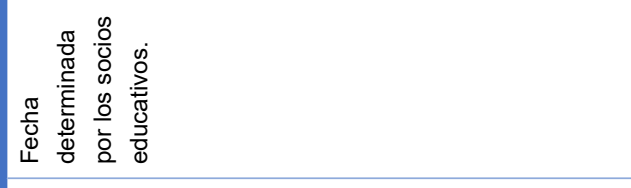 & 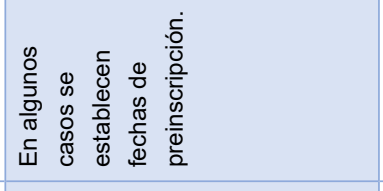 & \\
\hline 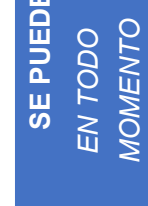 & 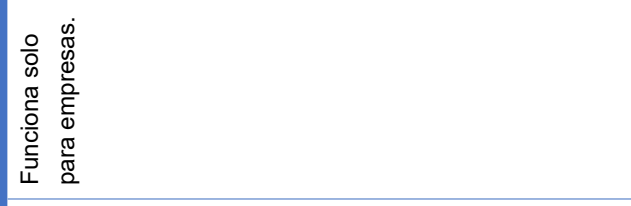 & & 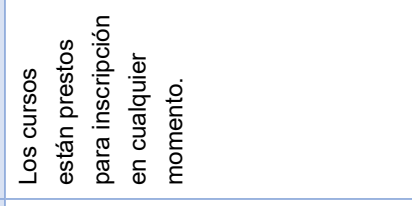 \\
\hline 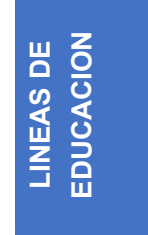 & 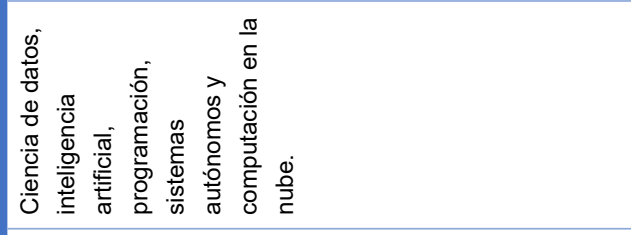 & 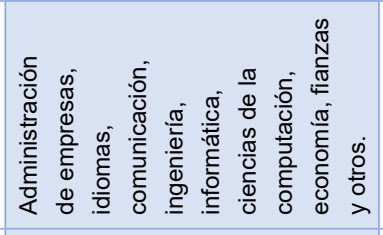 & 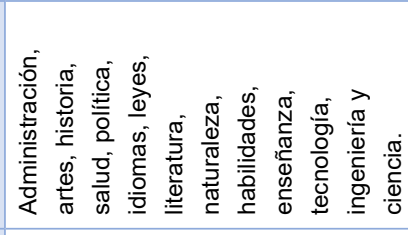 \\
\hline$\frac{2}{\circ}$ & 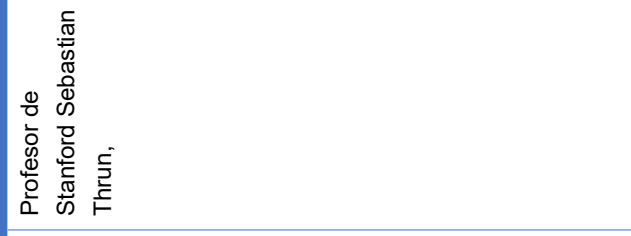 & 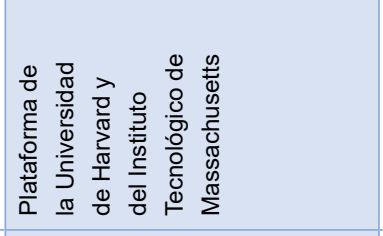 & 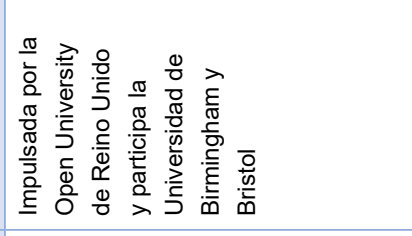 \\
\hline 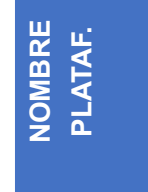 & 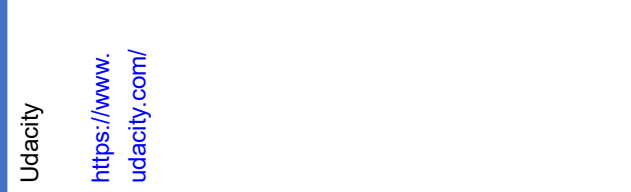 & 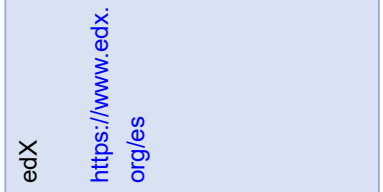 & 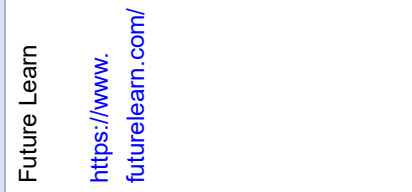 \\
\hline
\end{tabular}




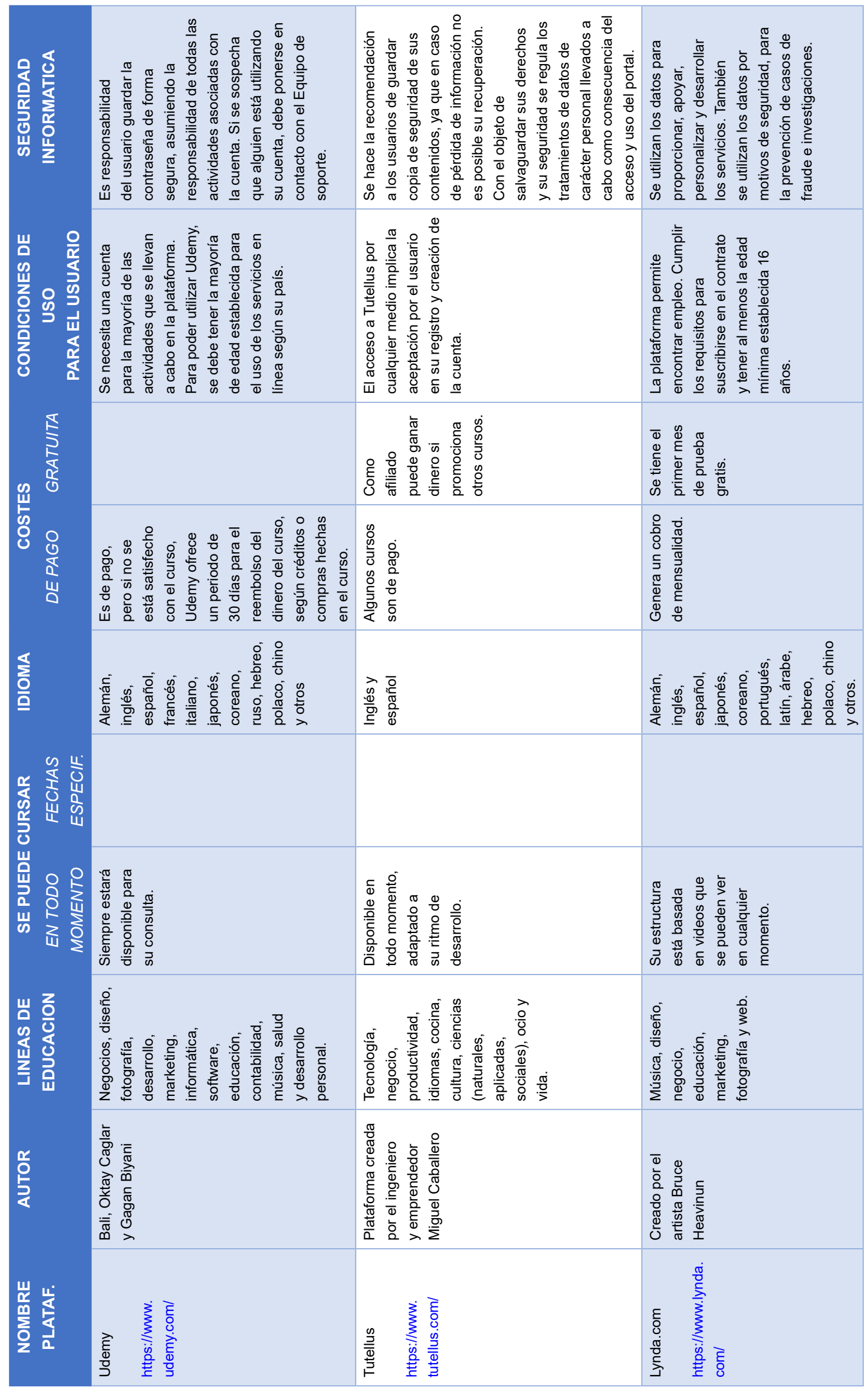




\begin{tabular}{|c|c|c|c|}
\hline 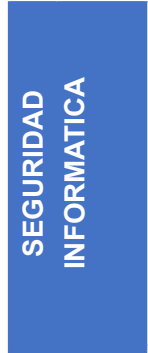 & 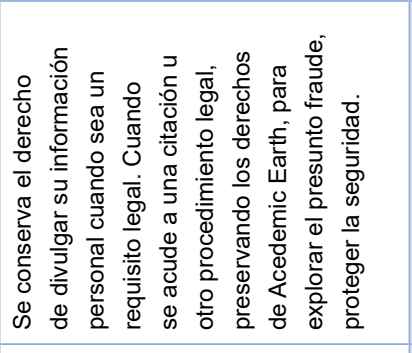 & 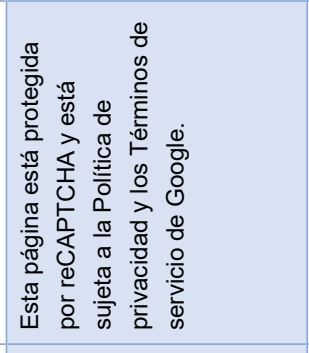 & 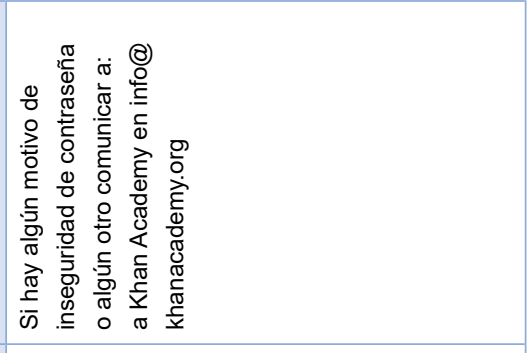 \\
\hline 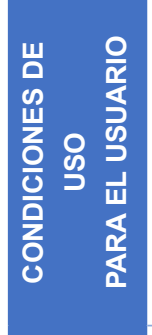 & 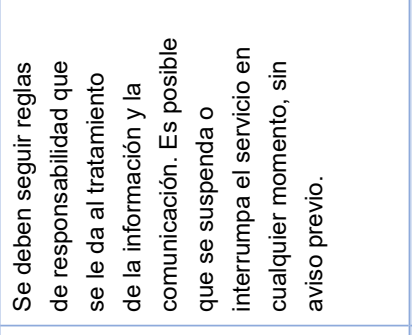 & 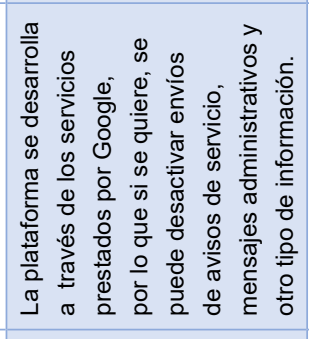 & 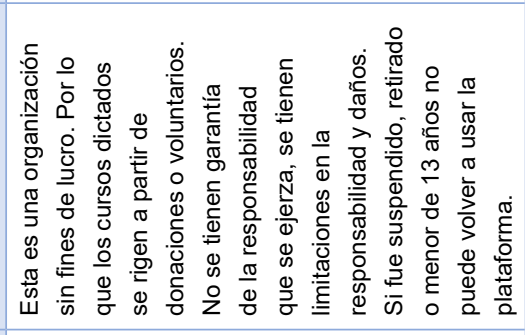 \\
\hline 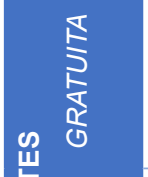 & 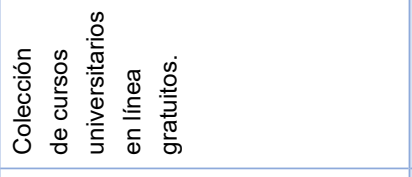 & & 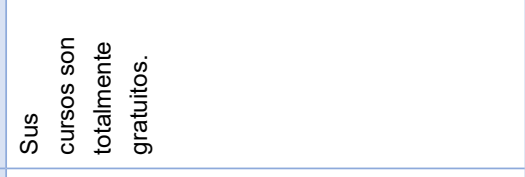 \\
\hline 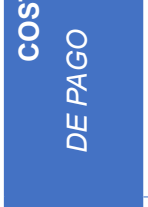 & & 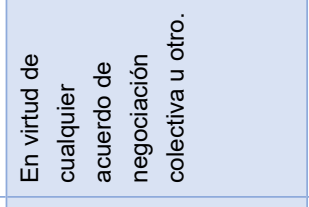 & \\
\hline $\begin{array}{l}\text { 동 } \\
\text { 음 }\end{array}$ & 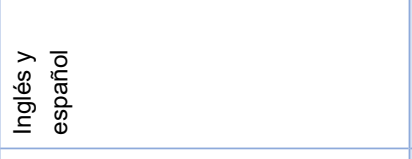 & $\begin{array}{l}\frac{\mathscr{\infty}}{\bar{m}} \\
\underline{\underline{\Xi}}\end{array}$ & $\begin{array}{l}\frac{\mathscr{n}}{9} \\
\underline{\underline{\mathscr{E}}}\end{array}$ \\
\hline 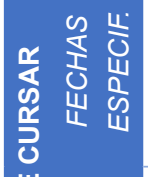 & & & \\
\hline 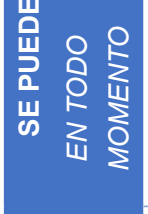 & 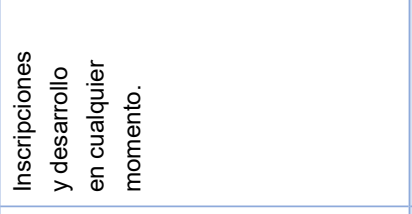 & 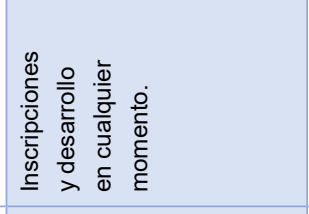 & 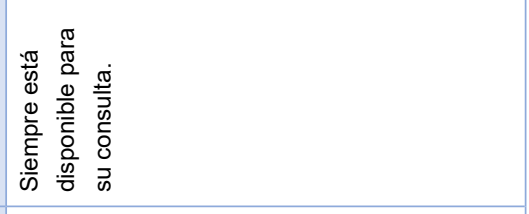 \\
\hline 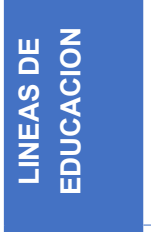 & 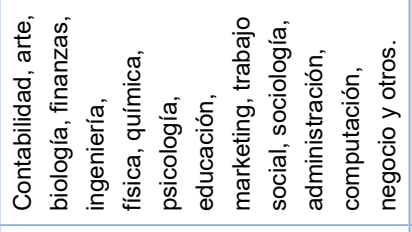 & 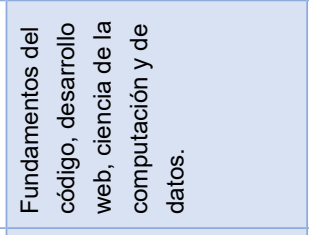 & 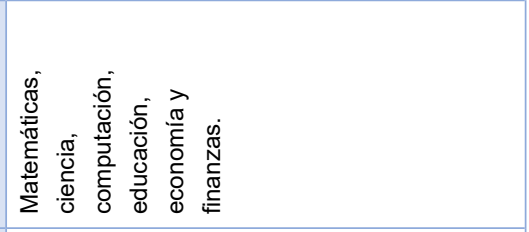 \\
\hline $\begin{array}{l}\frac{0}{0} \\
\frac{0}{5} \\
\frac{5}{4}\end{array}$ & 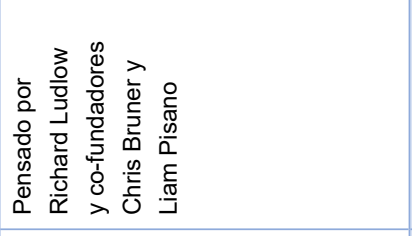 & 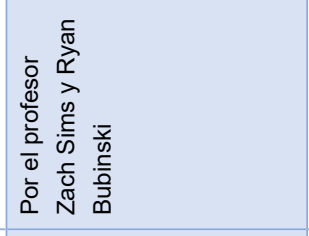 & 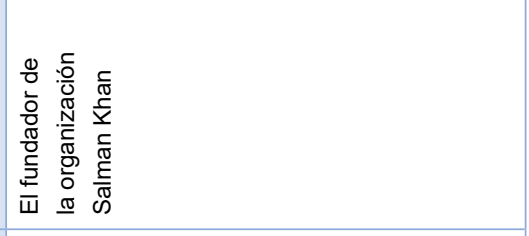 \\
\hline 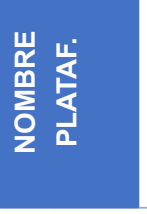 & 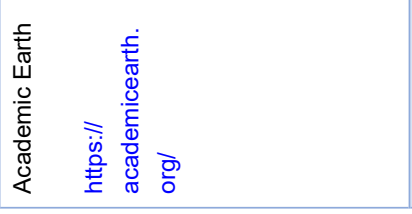 & 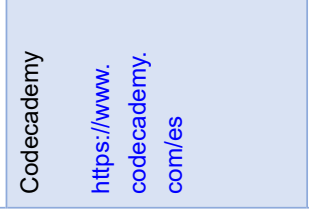 & 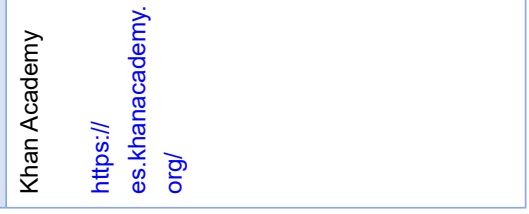 \\
\hline
\end{tabular}




\begin{tabular}{|c|c|c|c|}
\hline 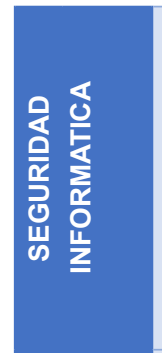 & 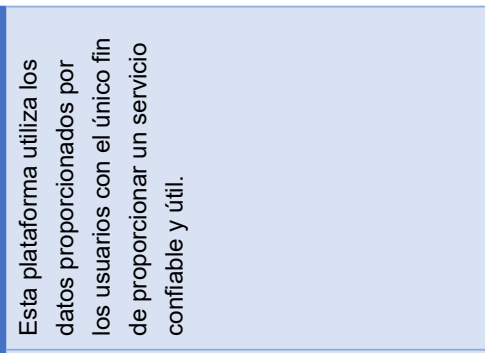 & 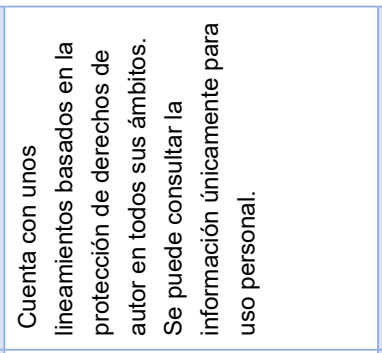 & 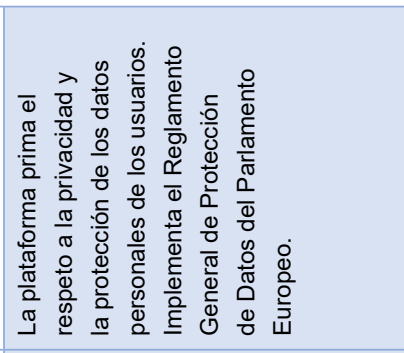 \\
\hline 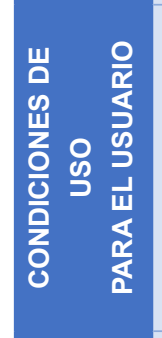 & 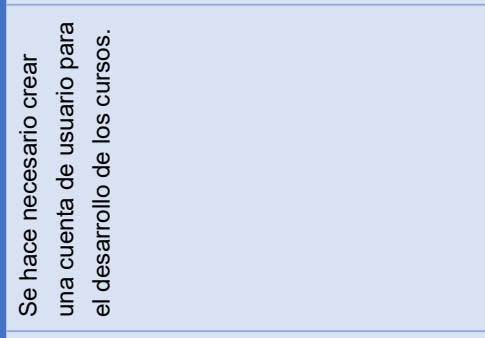 & 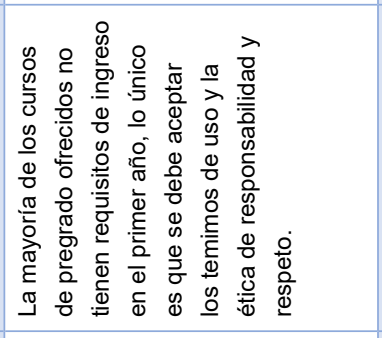 & 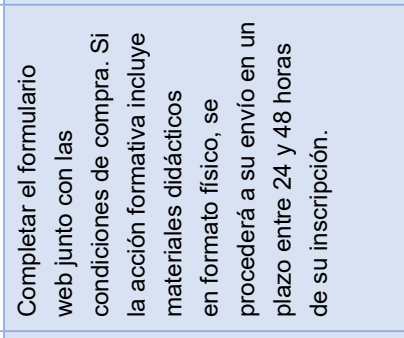 \\
\hline 点 & 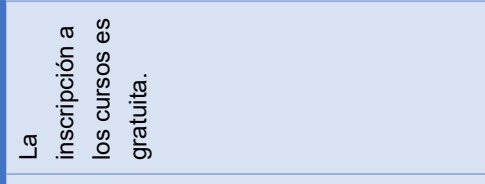 & 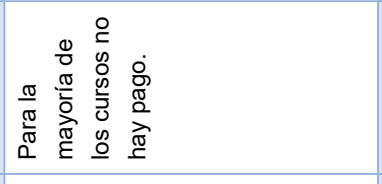 & \\
\hline 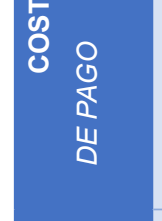 & 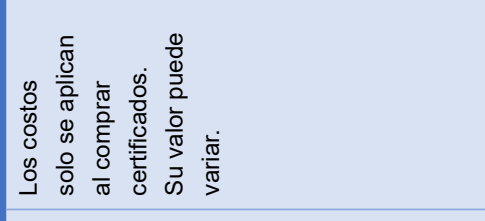 & 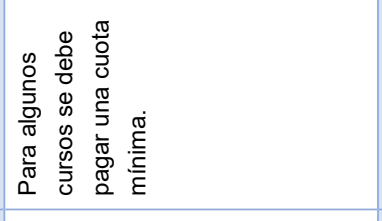 & 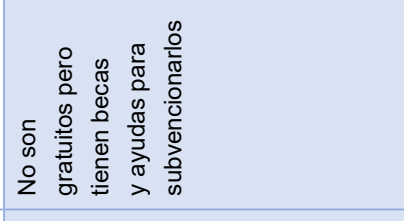 \\
\hline $\begin{array}{l}\text { 起 } \\
\text { 음 }\end{array}$ & 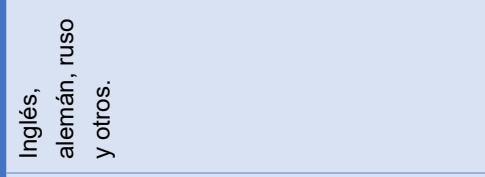 & $\begin{array}{l}\frac{\mathscr{D}}{0} \\
\underline{\underline{\underline{D}}}\end{array}$ & 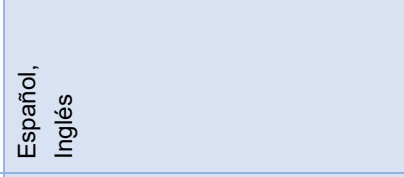 \\
\hline 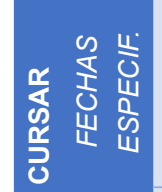 & & 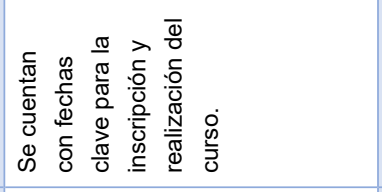 & \\
\hline 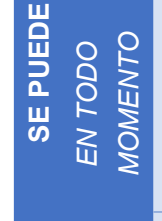 & 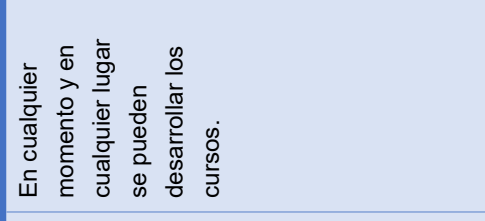 & & 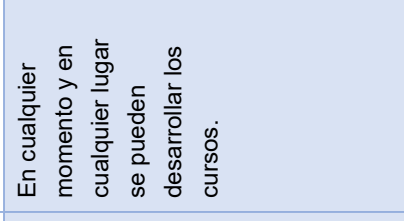 \\
\hline 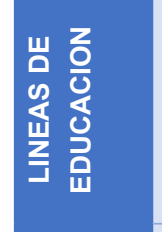 & 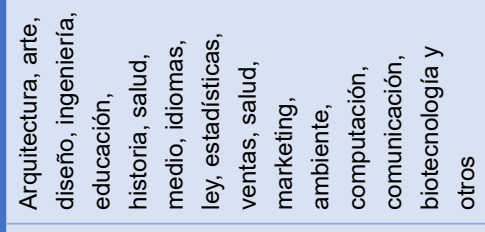 & 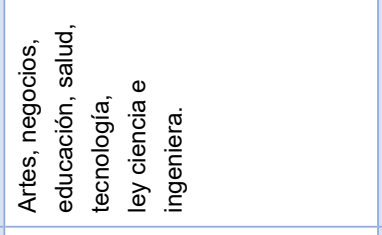 & 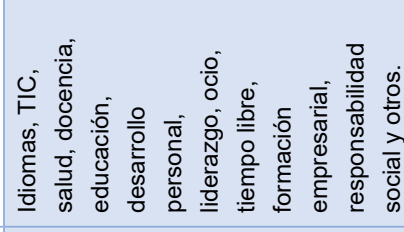 \\
\hline$\frac{c}{\circ}$ & 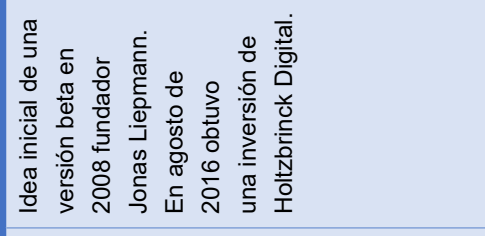 & 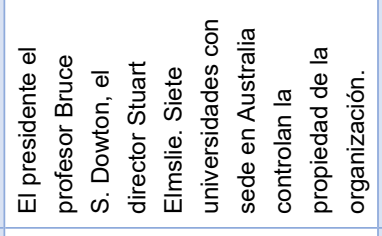 & 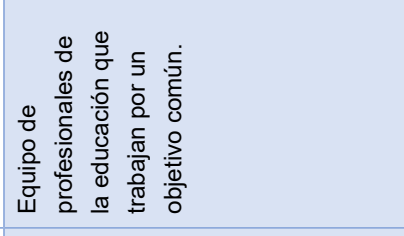 \\
\hline 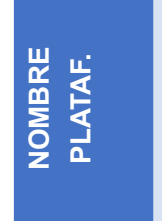 & 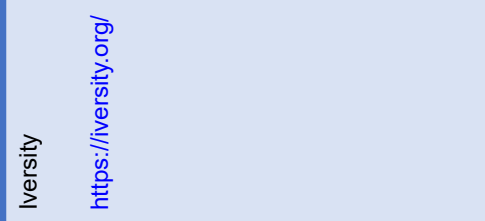 & 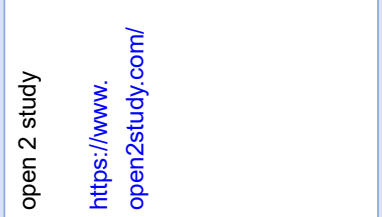 & 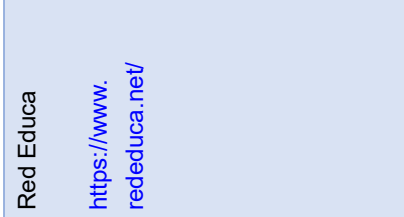 \\
\hline
\end{tabular}




\begin{tabular}{|c|c|c|c|c|}
\hline 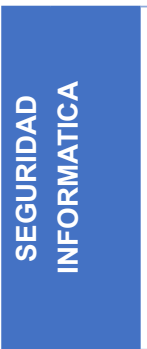 & 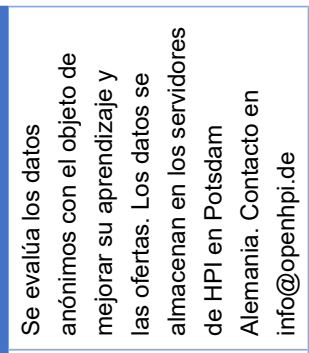 & 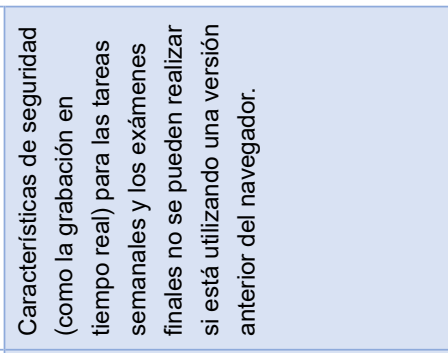 & 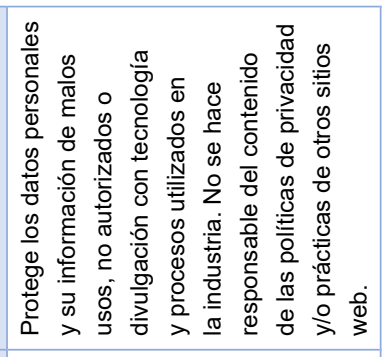 & 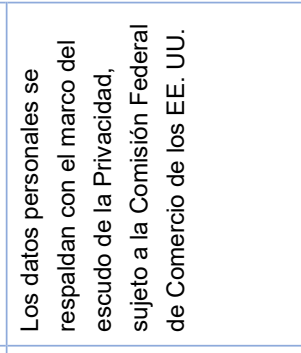 \\
\hline 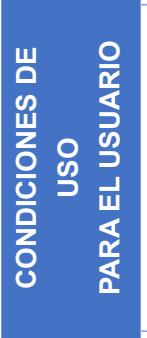 & 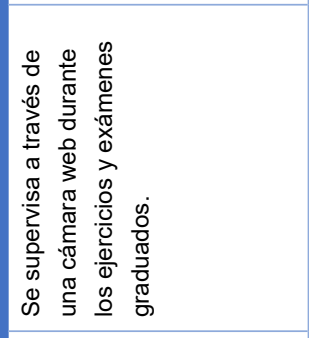 & 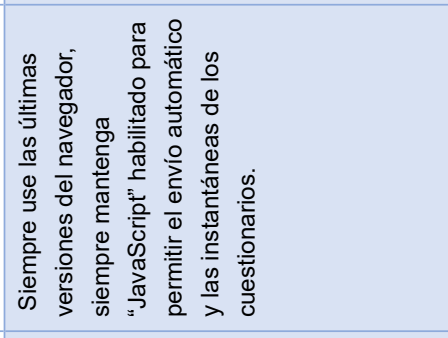 & 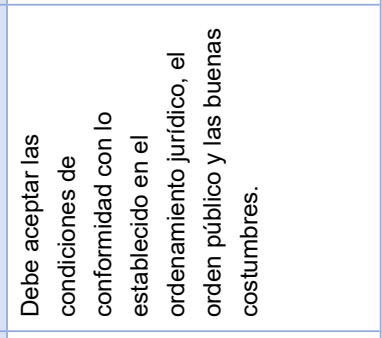 & 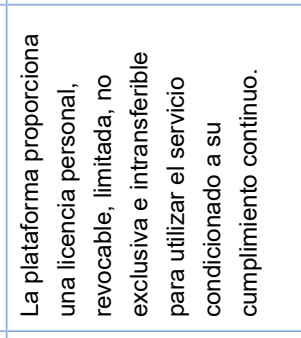 \\
\hline 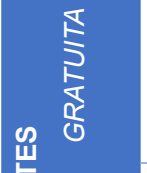 & 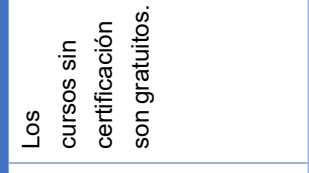 & 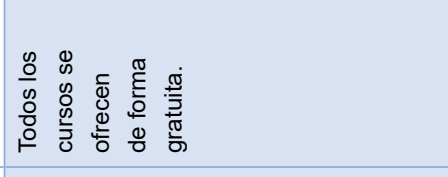 & & 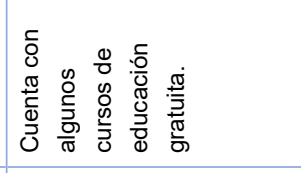 \\
\hline $\begin{array}{ll}8 & 0 \\
8 & 0 \\
0 & 0 \\
0 \\
0 \\
0\end{array}$ & 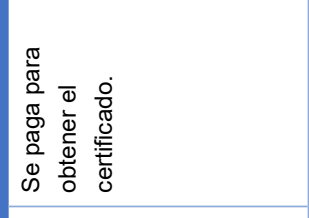 & 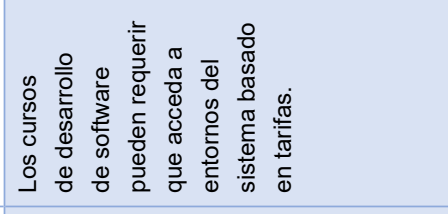 & 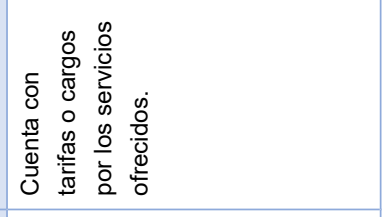 & 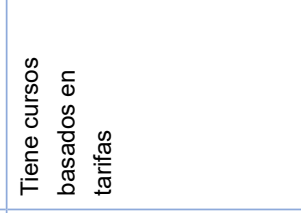 \\
\hline 음 & 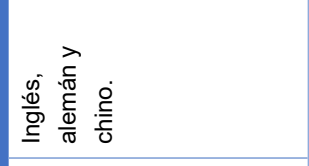 & 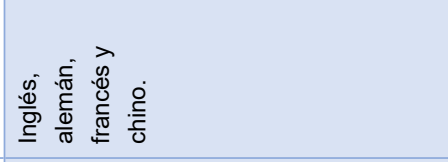 & 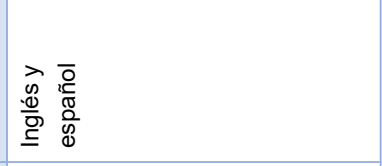 & 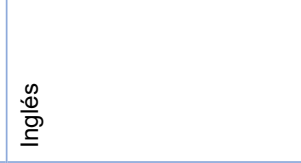 \\
\hline 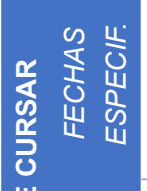 & 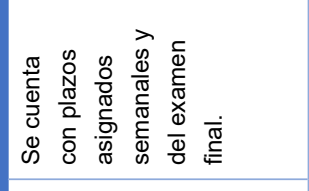 & 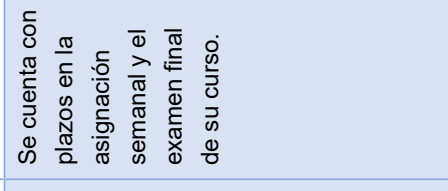 & & 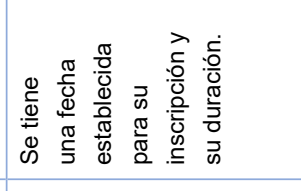 \\
\hline 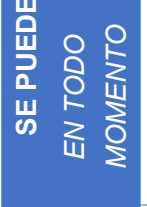 & 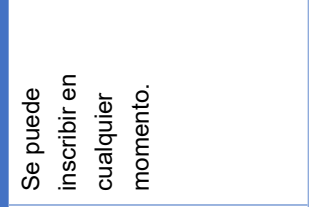 & 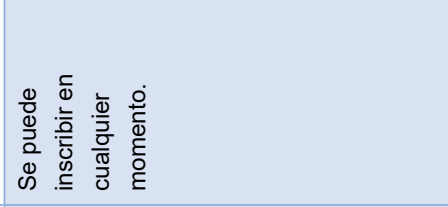 & 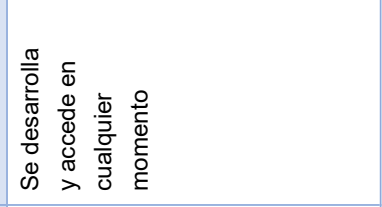 & \\
\hline 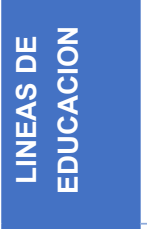 & 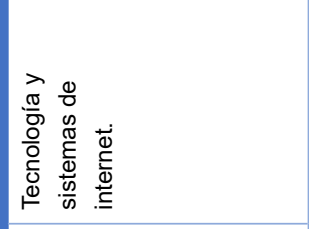 & 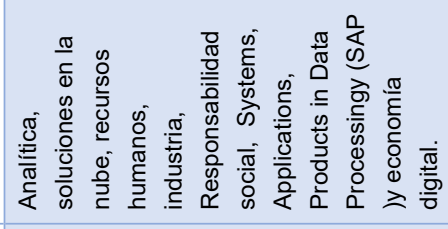 & 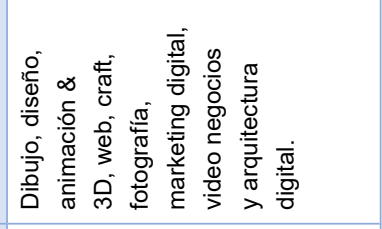 & 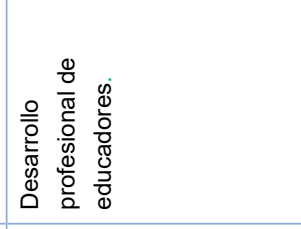 \\
\hline & 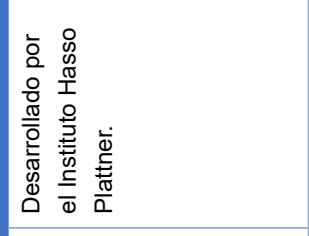 & 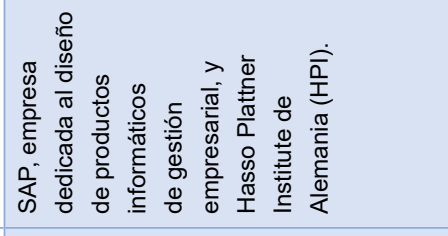 & 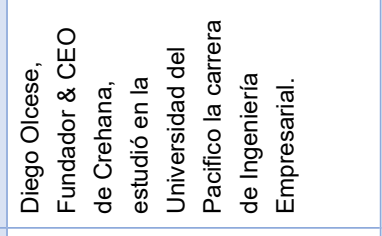 & 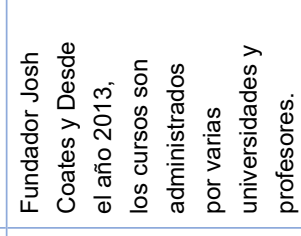 \\
\hline 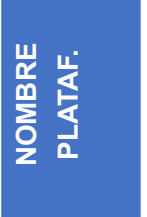 & 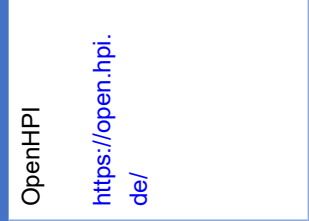 & 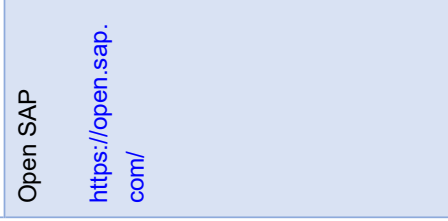 & 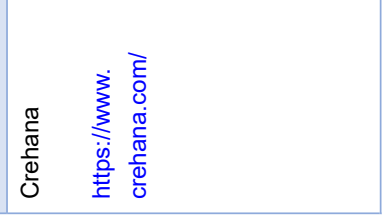 & 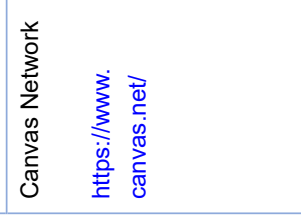 \\
\hline
\end{tabular}




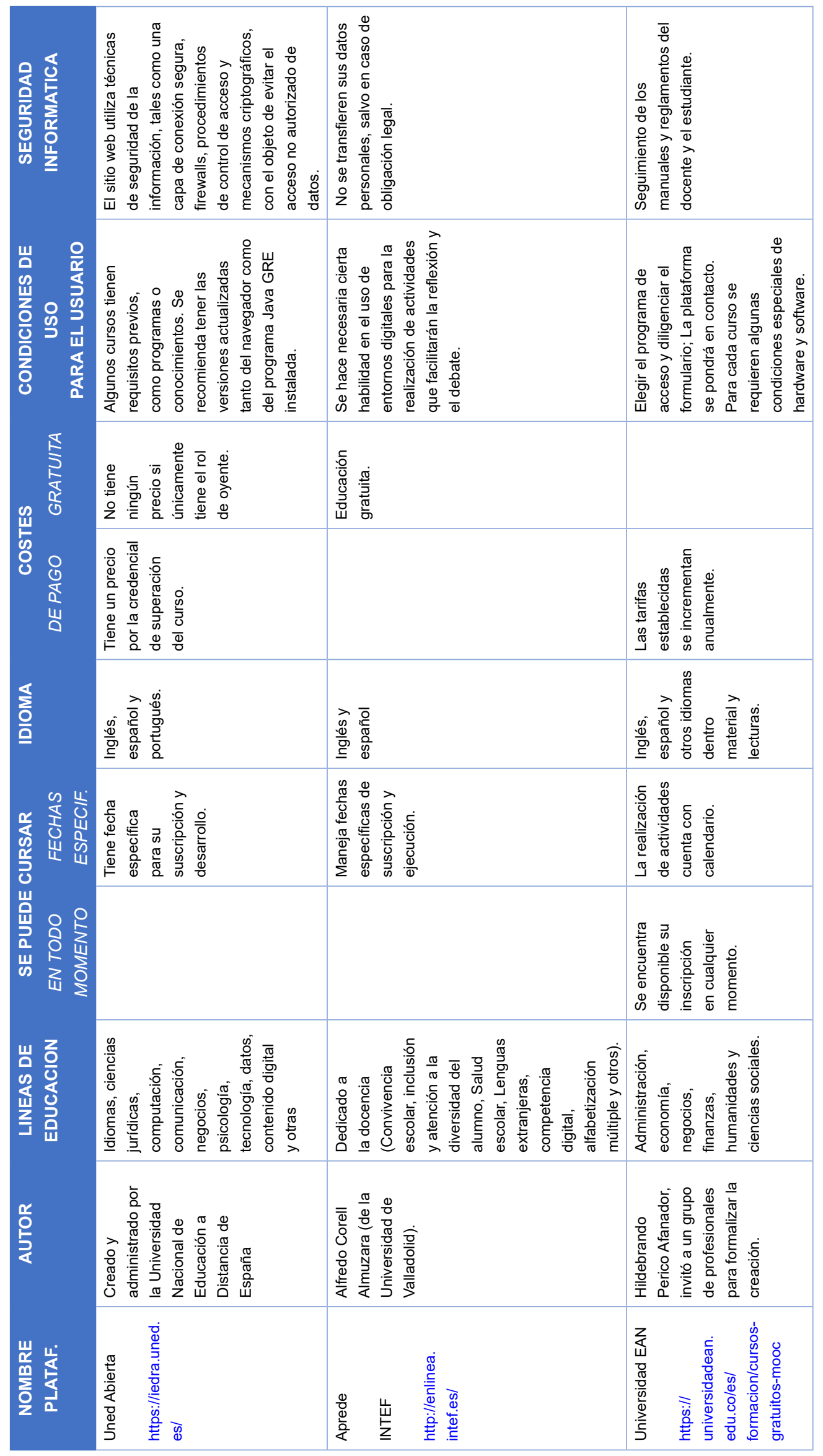




\begin{tabular}{|c|c|c|c|}
\hline 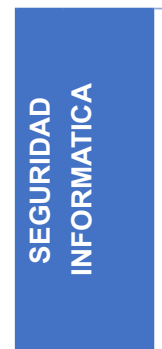 & 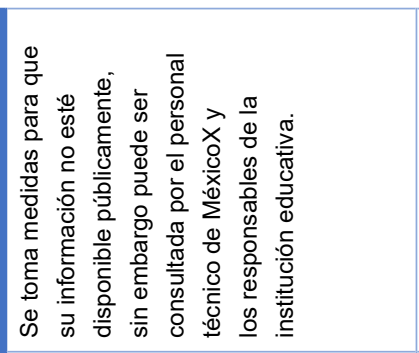 & 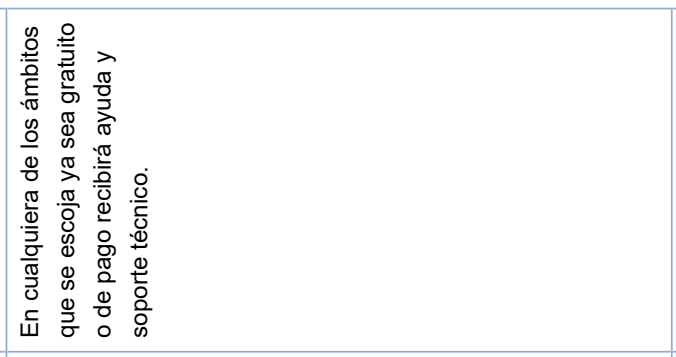 & 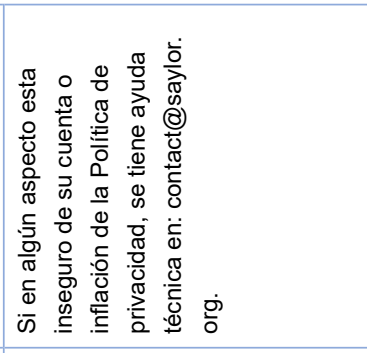 \\
\hline 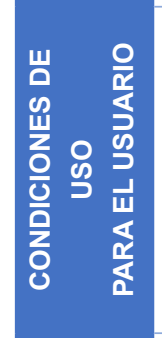 & 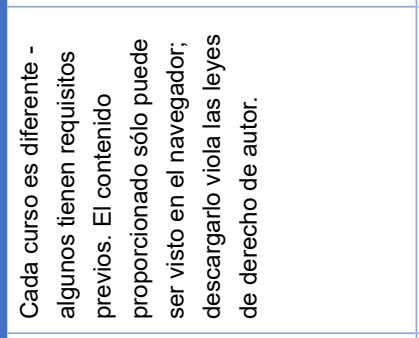 & 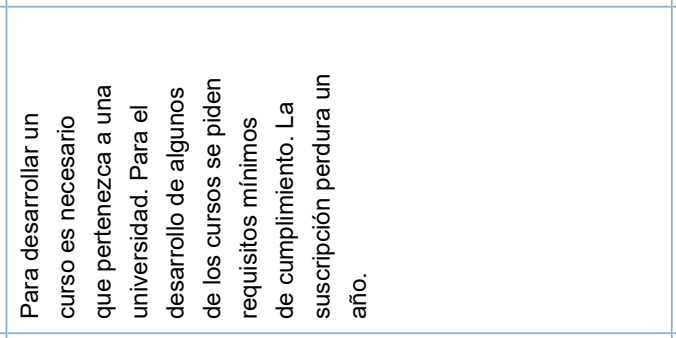 & 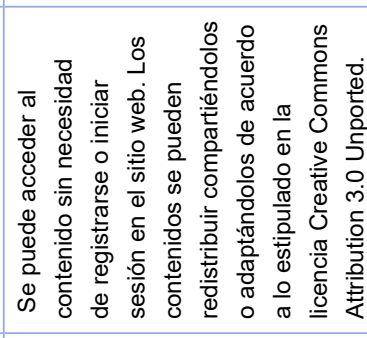 \\
\hline 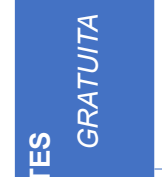 & 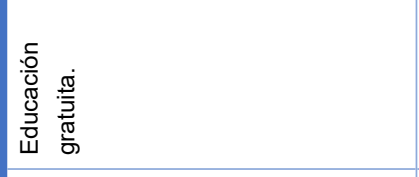 & 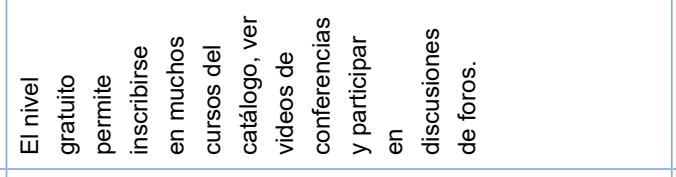 & 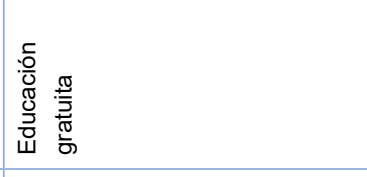 \\
\hline 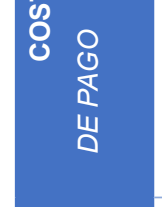 & & 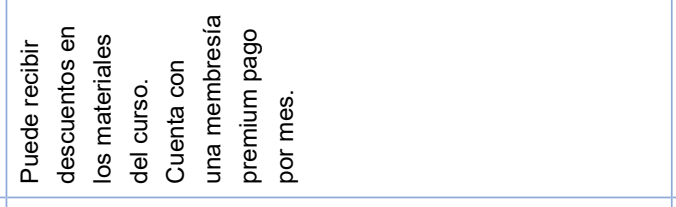 & \\
\hline & 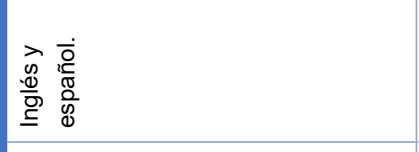 & 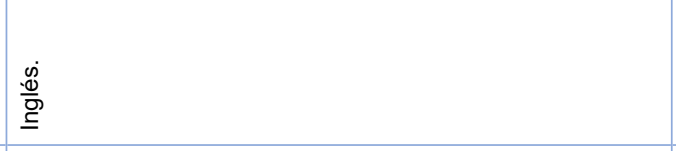 & 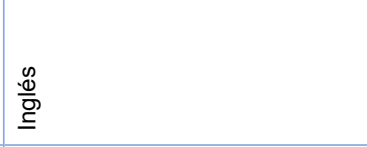 \\
\hline 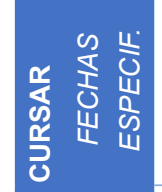 & 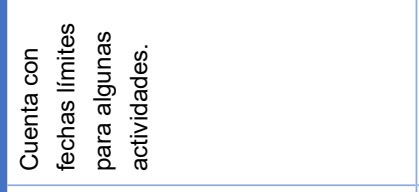 & 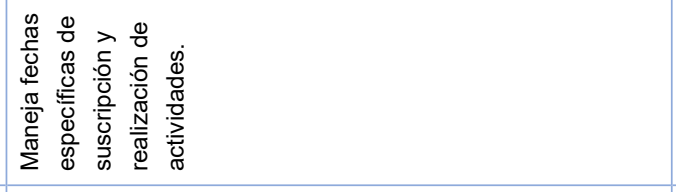 & \\
\hline 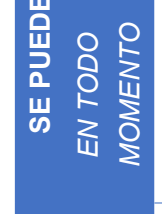 & 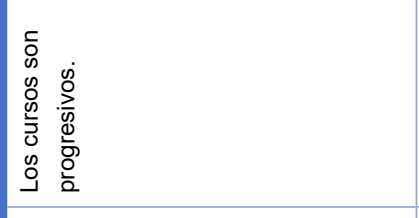 & & 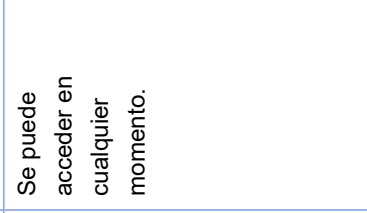 \\
\hline 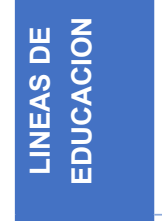 & 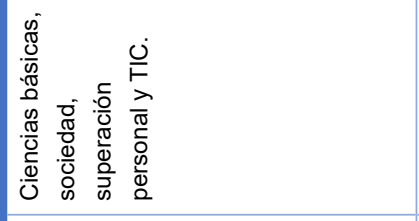 & 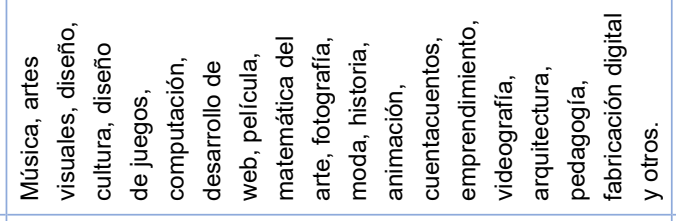 & 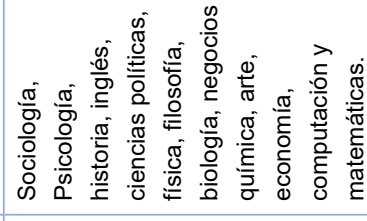 \\
\hline$\frac{\alpha}{\circ}$ & 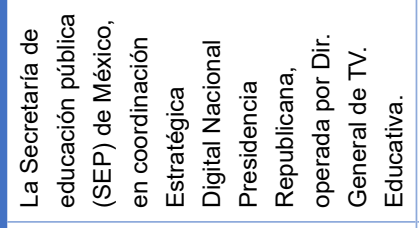 & 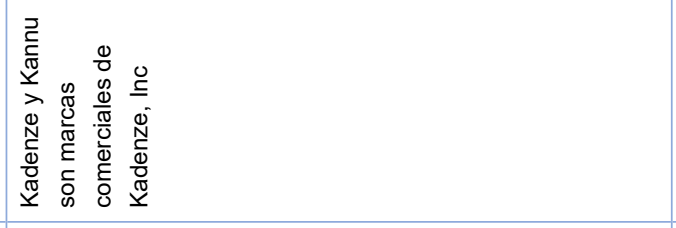 & 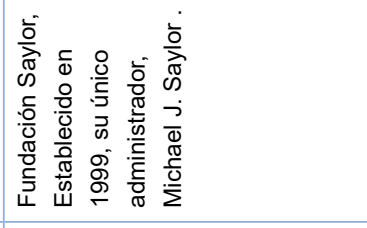 \\
\hline 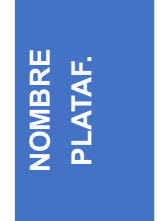 & 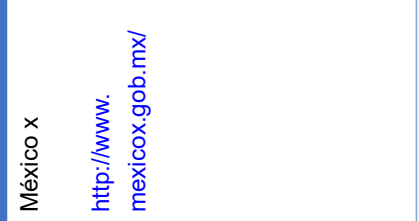 & 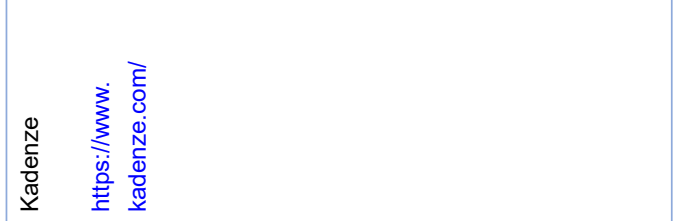 & 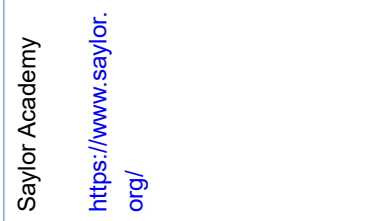 \\
\hline
\end{tabular}




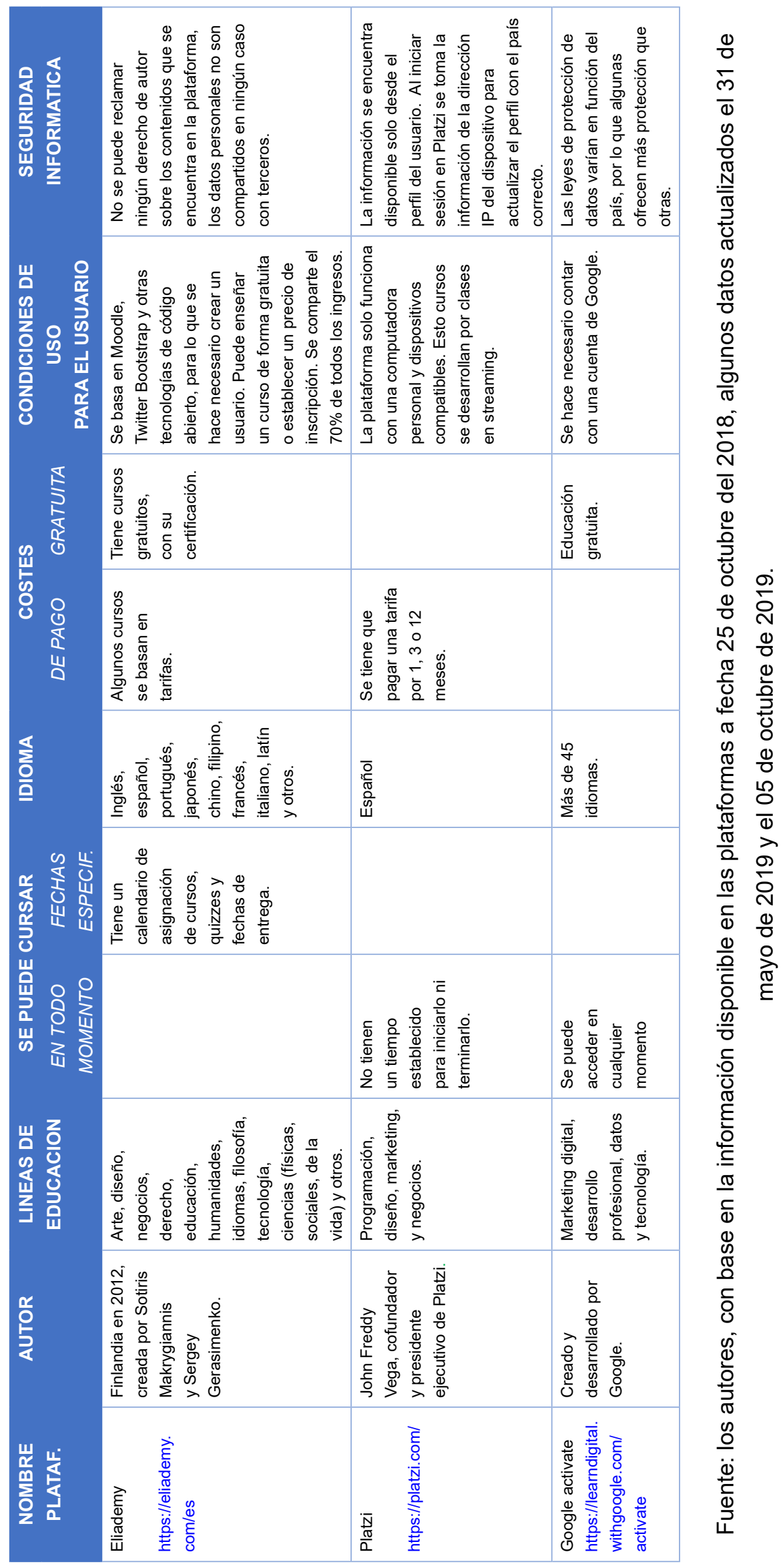




\section{PROPUESTA DE INDICADORES PARA LA SELECCIÓN DE PLATAFORMAS MOOC}

Para la gestión de desarrollo de tecnologías en las plataformas mencionadas, se consideró la toma de decisiones sobre enseñanza MOOC realizada por Peré (2017) y León (2014), donde se involucran los participantes, con diferentes implicaciones y en varias etapas del proceso, identificando la creación de recursos educativos y conocimiento abierto para la comunidad en general.

Con base en Zapata (2017), se hace inédita la necesidad de mejorar al mismo tiempo algunos tipos de tecnología, para medir la calidad según la innovación en un artefacto, por lo que considera tres aspectos tecnológicos: 1) tecnología de equipo, 2) tecnología de operación y 3) tecnología de producto. A partir de la estructuración anterior se plantean los indicadores tecnológicos, para la selección de plataformas de MOOC.

\section{TECNOLOGÍA DE EQUIPO}

Es el modo de manejo de herramientas e instrumentos para realizar tareas y de acuerdo con el DNP (2009, pág. 13), el protocolo o la metodología que cuantifica los recursos utilizados en el desarrollo de "la capacidad de acciones, procesos, procedimientos y operaciones realizadas durante la etapa de implementación".

\section{TECNOLOGÍA DE OPERACIÓN}

Es la capacidad informática del procedimiento para lograr un fin, que según el DNP (2009, pág. $13)$, en este caso mide los cambios resultantes en la observación y el análisis del "objetivo de la intervención como consecuencia (directa o indirecta) de la entrega del producto".

\section{TECNOLOGÍA DE PRODUCTO}

Es la técnica ligada a la utilidad con rendimiento y eficacia dada por el beneficio que según el DNP (2009, pág. 13), "cuantifica los bienes y servicios (intermedios o finales) producidos y/o provisionados a partir de una determinada intervención", así la información necesaria para desarrollar nuevos productos o servicios.

Dentro de las tres fases mencionadas se propusieron los indicadores tecnológicos según los siguientes aspectos:

Tabla 2:

Indicadores propuestos para los tres tipos de tecnología (equipo, operación y producto)

\begin{tabular}{|c|c|c|}
\hline INDICADOR & ASPECTO & DESCRIPCIÓN \\
\hline \multicolumn{3}{|c|}{ Tecnología de equipo } \\
\hline \multirow{3}{*}{$\begin{array}{l}\text { Notificaciones de } \\
\text { sucesos y eventos } \\
\text { de conexión }\end{array}$} & $\begin{array}{l}\text { Actualización } \\
\text { inmediata de } \\
\text { contenido }\end{array}$ & $\begin{array}{l}\text { Actualización inmediata y la programación de actualización para los } \\
\text { conjuntos de datos creados a partir de archivos. }\end{array}$ \\
\hline & $\begin{array}{l}\text { Reportes y } \\
\text { estadísticas }\end{array}$ & $\begin{array}{l}\text { Los usuarios de alguna manera tienen resumen de movimientos y } \\
\text { próximos vencimientos de actividades. }\end{array}$ \\
\hline & Automatización & $\begin{array}{l}\text { El acto que permite planificar y configurar de antemano, todos los } \\
\text { pasos involucrados en la realización del curso. }\end{array}$ \\
\hline $\begin{array}{l}\text { Opciones de } \\
\text { personalización }\end{array}$ & $\begin{array}{l}\text { Administración } \\
\text { y asignación de } \\
\text { permisos }\end{array}$ & $\begin{array}{l}\text { La definición de los permisos para editar y publicar, dentro de la } \\
\text { realización de un curso. }\end{array}$ \\
\hline
\end{tabular}




\begin{tabular}{|c|c|c|}
\hline $\begin{array}{l}\text { Herramientas de } \\
\text { comunicación. }\end{array}$ & $\begin{array}{l}\text { Medios de } \\
\text { comunicación }\end{array}$ & $\begin{array}{l}\text { En una plataforma MOOC es esencial que cuente con herramientas } \\
\text { de comunicación síncronas como (el Chat, la videoconferencia, } \\
\text { el MSN, entre otros) y herramientas de comunicación asíncronas } \\
\text { (como e-mail, redes sociales, entre otras). }\end{array}$ \\
\hline \multicolumn{3}{|c|}{ Tecnología de operación } \\
\hline \multirow[t]{2}{*}{$\begin{array}{l}\text { Compatibilidad } \\
\text { multiplataforma }\end{array}$} & $\begin{array}{l}\text { Ejecución de } \\
\text { hardware }\end{array}$ & Instrucciones específicas desde la misma consola. \\
\hline & $\begin{array}{l}\text { Ejecución de } \\
\text { software }\end{array}$ & $\begin{array}{l}\text { Los tipos de navegadores y programas adicionales, necesarios } \\
\text { para el buen funcionamiento de la plataforma. }\end{array}$ \\
\hline \multirow[b]{2}{*}{$\begin{array}{l}\text { Capacidad de } \\
\text { almacenamiento. }\end{array}$} & $\begin{array}{l}\text { Cantidad de datos } \\
\text { permitidos }\end{array}$ & $\begin{array}{l}\text { Medida estandarizada de la cantidad de datos que se pueden subir } \\
\text { y administrar en la red de una plataforma. }\end{array}$ \\
\hline & $\begin{array}{l}\text { Formatos } \\
\text { permitidos }\end{array}$ & $\begin{array}{l}\text { Tipos de archivo compatibles con la extensión de nombre de } \\
\text { archivo, según la plataforma. }\end{array}$ \\
\hline $\begin{array}{l}\text { Disponibilidad } \\
\text { de atención a los } \\
\text { requerimientos }\end{array}$ & Soporte técnico & $\begin{array}{l}\text { Si las plataformas MOOC dan solución a los usuarios sobre las } \\
\text { dudas, inquietudes y problemas, de manera satisfactoria. }\end{array}$ \\
\hline \multirow{2}{*}{$\begin{array}{l}\text { Necesidad de } \\
\text { software adicional } \\
\text { para el buen } \\
\text { funcionamiento del } \\
\text { MOOC. }\end{array}$} & $\begin{array}{l}\text { Requerimiento de } \\
\text { instalación }\end{array}$ & $\begin{array}{l}\text { Si la instalación o uso de la plataforma tiene alguna } \\
\text { incompatibilidad, espacio, costo u otro requisito para obtenerla. }\end{array}$ \\
\hline & $\begin{array}{l}\text { Mantenimiento del } \\
\text { aplicativo }\end{array}$ & $\begin{array}{l}\text { Cada cuanto hay necesidad de actualización del aplicativo y en que } \\
\text { costos adicionales infiere. }\end{array}$ \\
\hline \multicolumn{3}{|c|}{ Tecnología de producto } \\
\hline \multirow[b]{2}{*}{$\begin{array}{l}\text { Apoyo de uso al } \\
\text { usuario }\end{array}$} & Accesibilidad & $\begin{array}{l}\text { Máximo rango de usuarios que consultan una plataforma MOOC } \\
\text { en un mismo tiempo sin que se ponga lenta o se interrumpa la } \\
\text { conexión. }\end{array}$ \\
\hline & Usabilidad & $\begin{array}{l}\text { Entornos virtuales sencillos de usar por su facilidad de lectura } \\
\text { de los textos. Descarga rápida de información y presentación de } \\
\text { funciones y menús sencillos. }\end{array}$ \\
\hline $\begin{array}{l}\text { Existencia de } \\
\text { aplicación móvil } \\
(\mathrm{APP}) \text {. }\end{array}$ & APP & Si la plataforma MOOC cuenta con aplicativo móvil. \\
\hline \multirow{2}{*}{$\begin{array}{l}\text { Calidad de servicio } \\
\text { y producto de la } \\
\text { plataforma }\end{array}$} & \begin{tabular}{|l|} 
Servicios \\
adicionales
\end{tabular} & $\begin{array}{l}\text { Otros servicios prestados por en la plataforma diferentes a los } \\
\text { MOOC. }\end{array}$ \\
\hline & $\begin{array}{l}\text { Modos de } \\
\text { publicación }\end{array}$ & $\begin{array}{l}\text { Los usuarios pueden determinar los tiempos y modos de } \\
\text { publicación. }\end{array}$ \\
\hline $\begin{array}{l}\text { Soporte de } \\
\text { seguridad }\end{array}$ & $\begin{array}{l}\text { Seguridad } \\
\text { informática }\end{array}$ & $\begin{array}{l}\text { Que seguridad ofrecen las plataformas en cuanto a la información } \\
\text { que se incorpora dentro del proceso educativo. }\end{array}$ \\
\hline $\begin{array}{l}\text { Tasas de } \\
\text { abandono por } \\
\text { causas técnicas. }\end{array}$ & $\begin{array}{l}\text { Requerimientos } \\
\text { técnicos }\end{array}$ & $\begin{array}{l}\text { Los usuarios deben contar con requisitos básicos de hardware, } \\
\text { software y conexión a Internet. }\end{array}$ \\
\hline
\end{tabular}

Fuente: los autores con base en información recolectada dentro de la revisión de las plataformas MOOC. 


\section{RESULTADOS DEL ANÁLISIS DE LAS PLATAFORMAS Y LAS ENCUESTAS}

Se realizó el análisis de los requerimientos tecnológicos, en cuatro de las plataformas más utilizadas por los encuestados (Coursera, MiriadaX, edX y Udemy), como se muestra en la gráfica 1:

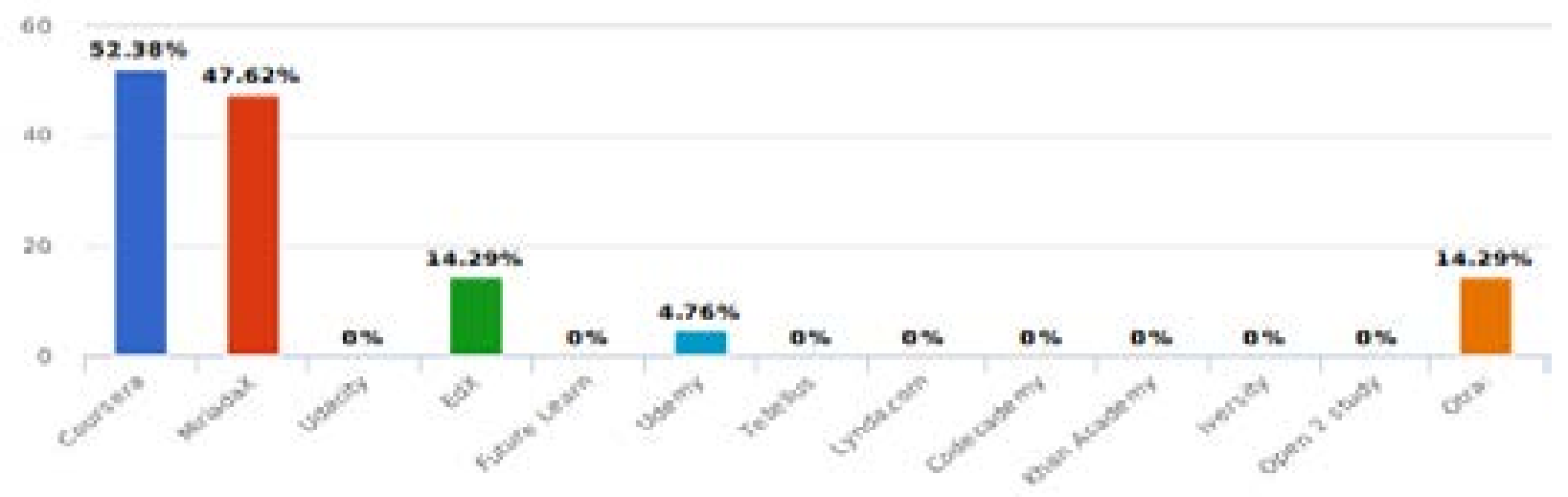

Grafica 1: Plataformas MOOC más usadas por los encuestados. Fuente: Los autores con base en las encuestas realizadas.

La revisión documental de las páginas web y la encuesta realizada, de acuerdo con los indicadores propuestos, arrojan los siguientes resultados:

\section{ANÁLISIS DE INDICADORES DE TECNOLOGÍA DE EQUIPO}

Se analizaron cada uno de los indicadores de la que se determinaron para el presente estudio, obteniendo la siguiente matriz:

Tabla 3: Análisis de Indicadores, Tecnología de equipo en las plataformas MOOC.

\begin{tabular}{|c|c|c|c|c|}
\hline & Coursera & MiríadaX & edX & Udemy \\
\hline $\begin{array}{l}\text { Notificaciones de } \\
\text { sucesos y eventos } \\
\text { de conexión }\end{array}$ & $\begin{array}{l}\text { Cuenta únicamente } \\
\text { con notificación } \\
\text { cuando el usuario } \\
\text { termina el curso. }\end{array}$ & $\begin{array}{l}\text { Notificación al correo } \\
\text { electrónico de las } \\
\text { actividades realizadas } \\
\text { y pendientes. }\end{array}$ & $\begin{array}{l}\text { No se reciben } \\
\text { notificaciones. }\end{array}$ & $\begin{array}{l}\text { Notificación por } \\
\text { correo electrónico. }\end{array}$ \\
\hline $\begin{array}{l}\text { Opciones de } \\
\text { personalización de } \\
\text { interfaces }\end{array}$ & $\begin{array}{l}\text { Se centra en } \\
\text { brindar una } \\
\text { estructura de guía } \\
\text { de videos, lecturas } \\
\text { y evaluación de la } \\
\text { unidad. }\end{array}$ & $\begin{array}{l}\text { Cuenta con una } \\
\text { estructura ya definida. }\end{array}$ & $\begin{array}{l}\text { Se cuenta con una } \\
\text { estructura definida } \\
\text { de videos, lecturas } \\
\text { y evaluaciones por } \\
\text { unidad. }\end{array}$ & $\begin{array}{l}\text { Crear acceso a } \\
\text { redes sociales y } \\
\text { personalizar el } \\
\text { logotipo, la imagen } \\
\text { de fondo y el icono } \\
\text { de la entidad. }\end{array}$ \\
\hline $\begin{array}{l}\text { Herramientas de } \\
\text { comunicación }\end{array}$ & $\begin{array}{l}\text { Blog, redes } \\
\text { sociales, correo } \\
\text { electrónico, } \\
\text { YouTube, entre } \\
\text { otros. }\end{array}$ & $\begin{array}{l}\text { Blog, correo } \\
\text { electrónico, foros de } \\
\text { discusión, chat, entre } \\
\text { otros. }\end{array}$ & $\begin{array}{l}\text { Foros de debate, blog, } \\
\text { correo electrónico, } \\
\text { foros de discusión, } \\
\text { wiki, entre otros. }\end{array}$ & $\begin{array}{l}\text { Blog, correo } \\
\text { electrónico y otros. }\end{array}$ \\
\hline
\end{tabular}

Fuente: los autores, con base en la información disponible en las plataformas el 25 de abril del 2019 y 19 de octubre de 2019. 
De acuerdo con lo anterior, la plataforma que se aprecia más completa en cuanto a características tecnológicas de equipo, es la plataforma Udemy, ya que tiene una variedad amplia de uso, manejo y desarrollo.
Por otro lado, para los usuarios encuestados, las herramientas de comunicación más utilizadas dentro del desarrollo de un MOOC, como se muestra en la gráfica 2:

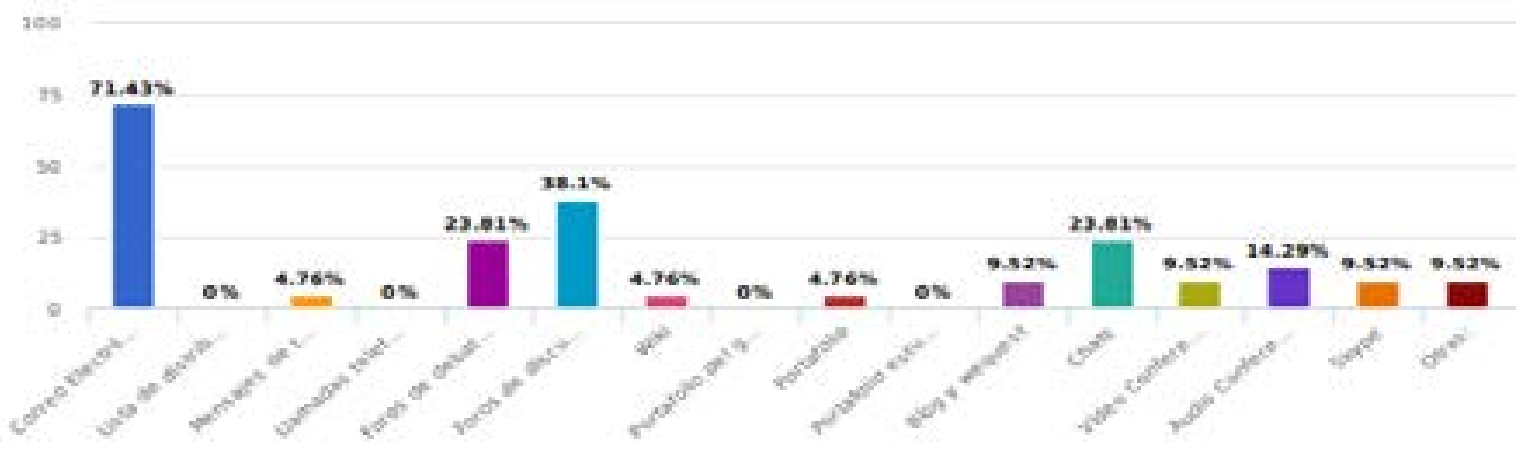

Grafica 2: Herramientas de comunicación. Fuente: Los autores con base en las encuestas realizadas

Herramientas de comunicación síncronas: se cuenta con chats, foros de debate $y$ comunicaciones académicas, para el $24 \%$ de los encuestados.

Herramientas de comunicación asíncronas: el más usado es el correo electrónico (con 71\%) y un segundo medio de comunicación son los foros de discusión (con 38\%).

\section{ANÁLISIS DE INDICADORES TECNOLOGÍA DE OPERACIÓN}

Se revisaron cada uno de los indicadores de la tecnología de operación en las plataformas MOOC que se determinaron para la presente continuación:

Tabla 4:

Análisis de Indicadores, Tecnología de operación en las plataformas MOOC.

\begin{tabular}{|c|c|c|c|c|}
\hline & Coursera & MiriadaX & edX & Udemy \\
\hline $\begin{array}{l}\text { Compatibilidad } \\
\text { con el navegador }\end{array}$ & $\begin{array}{l}\text { Funciona } \\
\text { adecuadamente en } \\
\text { el navegador google } \\
\text { Chrome y Mozilla. }\end{array}$ & $\begin{array}{l}\text { Se deben instalar } \\
\text { cookies (gestión } \\
\text { del registro, } \\
\text { Google Analytics, } \\
\text { Comscore, Score } \\
\text { Card Research, entre } \\
\text { otros) adicionales en } \\
\text { cada navegador }\end{array}$ & $\begin{array}{l}\text { Se deben instalar } \\
\text { cookies adicionales } \\
\text { en cada navegador. } \\
\text { No es compatible con } \\
\text { la última versión de } \\
\text { Mac }\end{array}$ & $\begin{array}{l}\text { Funciona } \\
\text { adecuadamente en } \\
\text { el navegador google } \\
\text { Chrome y Mozilla. }\end{array}$ \\
\hline
\end{tabular}




\begin{tabular}{|c|c|c|c|c|}
\hline $\begin{array}{l}\text { Capacidad de } \\
\text { almacenamiento }\end{array}$ & $\begin{array}{l}\text { No cuenta con } \\
\text { especificación de } \\
\text { almacenamiento. }\end{array}$ & $\begin{array}{l}\text { No se cuenta con } \\
\text { información. }\end{array}$ & $\begin{array}{l}\text { Todos los datos } \\
\text { se guardan en el } \\
\text { almacenamiento en la } \\
\text { nube de Amazon Web } \\
\text { Services. }\end{array}$ & $\begin{array}{l}\text { El material se puede } \\
\text { cargar por lotes o } \\
\text { importarlos a través } \\
\text { de Google Drive, } \\
\text { Box, Dropbox, entre } \\
\text { otras. }\end{array}$ \\
\hline $\begin{array}{l}\text { Disponibilidad } \\
\text { de atención a los } \\
\text { requerimientos }\end{array}$ & $\begin{array}{l}\text { La respuesta a un } \\
\text { requerimiento no } \\
\text { tarda más de una } \\
\text { hora. }\end{array}$ & $\begin{array}{l}\text { Se solicitó } \\
\text { información al } \\
\text { respecto a la } \\
\text { plataforma, pero no } \\
\text { se obtuvo respuesta. }\end{array}$ & $\begin{array}{l}\text { Se atiende a los } \\
\text { requerimientos } \\
\text { únicamente en días } \\
\text { laborables. }\end{array}$ & $\begin{array}{l}\text { Se solicitó } \\
\text { información al } \\
\text { respecto a la } \\
\text { plataforma, pero } \\
\text { no se obtuvo } \\
\text { respuesta. }\end{array}$ \\
\hline $\begin{array}{l}\text { Necesidad } \\
\text { de software } \\
\text { adicional }\end{array}$ & $\begin{array}{l}\text { Se usan herramientas } \\
\text { de terceros para } \\
\text { modificar la } \\
\text { plataforma. }\end{array}$ & $\begin{array}{l}\text { En caso de que sea } \\
\text { necesario algún } \\
\text { requisito, el instructor } \\
\text { lo notifica. }\end{array}$ & $\begin{array}{l}\text { Se usan herramientas } \\
\text { de terceros en } \\
\text { beneficio de la } \\
\text { plataforma. }\end{array}$ & $\begin{array}{l}\text { Se cuenta con } \\
\text { herramientas } \\
\text { de terceros en } \\
\text { Marketing y } \\
\text { comunicación. }\end{array}$ \\
\hline
\end{tabular}

Fuente: los autores, con base en la información disponible en las plataformas el 25 de abril del 2019 y 19 de octubre de 2019.

En varias de las plataformas MOOC, se solicita la instalación obligatoria de Java Script, además de contar con un navegador e internet, de ser necesario otro tipo de programa para el desarrollo mismo del curso, este es notificado por el instructor a cargo.

Según el análisis, la plataforma que ofrece mejores indicadores tecnológicos de operación, es edX, ya que cuenta con alta capacidad de almacenamiento, respuesta rápida y operación con diversidad de soluciones prestadas por terceros.

Por otro lado, se tiene que en un $62 \%$ de los encuestados, que realizaron MOOC dentro de las plataformas mencionadas, no tuvieron inconvenientes a la hora de subir archivos, por lo que la capacidad de almacenamiento en cualquiera de las plataformas es apta para las condiciones necesarias de un curso. Cuando se presentaron errores en mayoría, fue por falla de la conexión que dependía del usuario.

\section{Análisis de los Indicadores Tecnología de producto}

Cada uno de los indicadores de la tecnología de producto en las plataformas MOOC estudiadas, se analizan a continuación: 
Tabla 5:

Análisis de Indicadores, Tecnología de producto en las plataformas MOOC.

\begin{tabular}{|c|c|c|c|c|}
\hline & Coursera & MiriadaX & edX & Udemy \\
\hline Ayuda de uso & $\begin{array}{l}\text { Se cuenta con una } \\
\text { interfaz con todas } \\
\text { las respuestas de } \\
\text { preguntas realizadas } \\
\text { por antiguos usuario. }\end{array}$ & $\begin{array}{l}\text { Se cuenta con un } \\
\text { link de preguntas } \\
\text { frecuentes. }\end{array}$ & $\begin{array}{l}\text { Interfaz con todas } \\
\text { las respuestas de } \\
\text { preguntas generadas } \\
\text { por usuarios } \\
\text { anteriores. }\end{array}$ & $\begin{array}{l}\text { Interfaz con todas } \\
\text { las respuestas de } \\
\text { preguntas realizadas } \\
\text { anteriormente. }\end{array}$ \\
\hline$A p p$ & $\begin{array}{l}\text { Cuenta con app } \\
\text { (tiene un peso de } \\
39 \mathrm{MB} \text {, puntuación } \\
\text { de } 4.4 \text { y más de } 5 \mathrm{M} \\
\text { descargas). }\end{array}$ & $\begin{array}{l}\text { Cuenta con app } \\
\text { (tiene un peso de } 10 \\
\text { MB, puntuación de } \\
3.2 \text { y más de } 50 \mathrm{M} \\
\text { descargas). }\end{array}$ & $\begin{array}{l}\text { Cuenta con app } \\
\text { (tiene un peso de } \\
10 \mathrm{MB} \text {, puntuación } \\
\text { de } 4.6 \text { y más de } 1 \mathrm{M} \\
\text { descargas). }\end{array}$ & $\begin{array}{l}\text { Cuenta con app, } \\
\text { (tiene un peso de } \\
11 \mathrm{MB} \text {, puntuación } \\
\text { de } 4.5 \text { y más de } 5 \mathrm{M} \\
\text { descargas). }\end{array}$ \\
\hline $\begin{array}{l}\text { Calidad del } \\
\text { servicio y } \\
\text { productos de la } \\
\text { plataforma }\end{array}$ & $\begin{array}{l}\text { Adaptaciones para } \\
\text { estudiantes con } \\
\text { deficiencias auditivas, } \\
\text { visuales y de } \\
\text { aprendizaje. }\end{array}$ & $\begin{array}{l}\text { No se pudo obtener } \\
\text { información. }\end{array}$ & $\begin{array}{l}\text { No se pudo obtener } \\
\text { información. }\end{array}$ & $\begin{array}{l}\text { No se pudo obtener } \\
\text { información. }\end{array}$ \\
\hline $\begin{array}{l}\text { Soporte técnico } \\
\text { y Seguridad }\end{array}$ & $\begin{array}{l}\text { Se usa la información } \\
\text { personal identificable } \\
\text { para el soporte técnico } \\
\text { y para la seguridad de } \\
\text { los servicios y el sitio } \\
\text { en todo momento. }\end{array}$ & $\begin{array}{l}\text { Se cuenta con } \\
\text { soporte únicamente } \\
\text { si tiene problemas } \\
\text { con el pago o alguna } \\
\text { condición de usuario } \\
\text { que falle. }\end{array}$ & $\begin{array}{l}\text { Chats en vivo, que } \\
\text { de no contar con } \\
\text { la respuesta se } \\
\text { comunicaran luego } \\
\text { dejando el correo } \\
\text { electrónico. }\end{array}$ & $\begin{array}{l}\text { Si se infringe alguna } \\
\text { de las políticas de } \\
\text { Udemy puede, que } \\
\text { por un tiempo este } \\
\text { penalizado y no } \\
\text { pueda entrar a la } \\
\text { plataforma. }\end{array}$ \\
\hline $\begin{array}{l}\text { Tasa de } \\
\text { abandono }\end{array}$ & $\begin{array}{l}\text { Entre el } 95 \% \text { y } 96 \% \\
\text { no terminan el curso, } \\
\text { Collazos }(2014) .\end{array}$ & $\begin{array}{l}\text { Entre un } 90 \% \text { y } \\
92 \% \text { no terminan los } \\
\text { cursos. }\end{array}$ & $\begin{array}{l}\text { No se cuenta con } \\
\text { información al } \\
\text { respecto. }\end{array}$ & $\begin{array}{l}\text { Promedio del } 30 \% \\
\text { no termina el curso. }\end{array}$ \\
\hline
\end{tabular}

Fuente: los autores, con base en la información disponible en las plataformas el 25 de abril del 2019 y 19 de octubre de 2019.

La plataforma que se destaca en los indicadores de tecnología de producto es Coursera, puesto que cuenta con adaptación para discapacidades audiovisuales. En cuanto a la app, la plataforma edX es la que cuenta con mayor puntuación de calificación, aunque es la de menos descargas generadas.
Como resultado de las encuestas se tiene una tasa de abandono la cual es de un $28 \%$, a causa de: el tiempo, el desinterés y el pago de la inscripción de la plataforma o certificación. Por el contrario, todos los encuestados están interesados en volver a cursar un MOOC. 


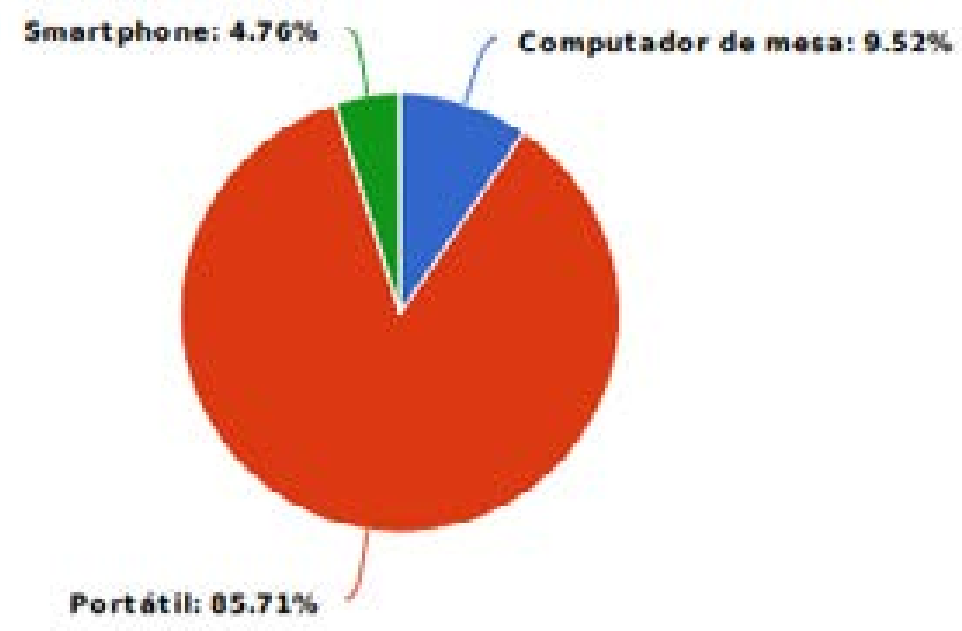

Grafica 3: Accesorios tecnológicos utilizados para el desarrollo de un curso. Fuente: Los autores con base en las encuestas realizadas

Como se muestra en la gráfica 3. El dispositivo más usado para realizar un MOOC con un $86 \%$ es el computador portátil, y en un $5 \%$ con Smartphone, ya que se manifiesta que no todas las plataformas cuentan con aplicativo móvil y es más complejo su uso.

\section{DISCUSIÓN}

Al realizar una perspectiva cruzada entre lo mencionado por los autores y los resultados previamente presentados en relación al análisis de cada uno de los aspectos tecnológicos concernientes con los MOOC, las plataformas que los ofrecen y las encuestas realizadas, se puede traer a colación ciertos indicadores que permiten medir la calidad de un artefacto, y que desde la perspectiva de Zapata (2017) estos son divididos en tres aspectos tecnológicos: 1) tecnología de equipo, 2) tecnología de operación y 3) tecnología de producto.

En adición a lo mencionado por zapata y para el abordaje de este ítem de discusión, se hace necesario también tener en cuenta lo mencionado por DNP (2009) y que fue citado preliminarmente para cada uno de los tres indicadores.

\section{- 1) INDICADORES DE TECNOLOGÍA \\ DE EQUIPO}

Tomando lo mencionado por Zapata (2017) y por la DNP (2009, pág. 13) y en contraste con lo encontrado en los resultados, se hizo evidente la capacidad de las acciones, procesos, procedimientos y operaciones, aunque debe aclararse que estas capacidades se hicieron más notorias en relación a la plataforma Udemy en el uso, manejo y desarrollo; tanto de las notificaciones, como de las opciones de personalización de la interfaz, así como de las herramientas de comunicación.

En cuanto a lo manifestado en las encuestas, las herramientas de comunicación en las operaciones de la etapa de implementación de los distintos cursos por parte de las plataformas: de manera síncronas se contó con un mayor uso de chats, foros de debate, así como de comunicaciones académicas; y de manera asíncronas las herramientas más usadas son el correo electrónico y los foros de discusión como segunda alternativa. 


\section{- 2) INDICADORES DE TECNOLOGÍA DE OPERACIÓN}

En lo que respecta a los indicadores de tecnología de operación y al enfrentar lo ya mencionado por Zapata (2017) y por la DNP (2009, pág. 13) con los resultados, se pudo observar que si bien se da una medición de los cambios resultantes en relación a la intervención directa o indirecta, según el análisis realizado la plataforma que brinda mejores indicadores tecnológicos de operación es edX esto dado de manera indirecta por la diversidad de soluciones prestadas por terceros y de manera directa con la capacidad de almacenamiento además de respuesta rápida en la solución de los requerimientos.

Respectivamente y en concordancia con lo mencionado previamente, un buen porcentaje de los encuestados no tuvieron inconvenientes a la hora de subir archivos, además de que cuando se presentaron errores estos se dieron por una falla en la conexión por parte del usuario y no por parte de la plataforma.

\section{- 3) INDICADORES DE TECNOLOGÍA DE PRODUCTO}

En lo que concierne a los indicadores de tecnología de producto y al contraponer lo sustentado por Zapata (2017) y por la DNP (2009, pág. 13) con los resultados, se consiguió establecer que en lo tecnológico las plataformas que mejor cuantificados tienen sus servicios en relación a la utilidad, su rendimiento y eficacia, son por un lado Coursera el cual cuenta con una mejor adaptación a las discapacidades audiovisuales, y por otro lado y en relación a la app edX que cuenta con mayor puntuación por parte de los usuarios, aunque es la que menos descargas generadas tiene.

\section{CONCLUSIONES}

Toda plataforma cuenta con características diferentes en lo que respecta a tecnología, estas se centran en diferentes tipos de usuarios, por lo que es conveniente para una universidad que quiera brindar educación de manera virtual, la selección de la plataforma MOOC que se adecuen a sus necesidades, el reto está en definir los componentes tecnológicos requeridos de acuerdo a la estructura y forma que se quiera dar el curso, y teniendo en cuenta la población hacia la que se enfoca.

La medición de los componentes tecnológicos con las que cuenta cada plataforma es esencial, ya que entre más capacidad de tecnología se preste, más posibilidades de que los instructores empleen actividades y contenidos con programas más profesionales, por lo que la propuesta de indicadores aquí presentados se centra en tres aspectos importantes para la selección de una plataforma adecuada a la educación que se quiera brindar.

Esta propuesta evaluó la calidad de las plataformas MOOC mediante componentes tecnológicos, presentado una propuesta de indicadores divididos en tres clases (tecnología de equipo, tecnología de operación y tecnología de producto), estudiadas y valoradas por una muestra de encuestados. Los indicadores cuantifican y cualifican los aspectos tecnológicos encontrados dentro de las plataformas MOOC, como método de medición de la capacidad de acciones, procesos, procedimientos, operaciones, cambios, bienes y/o servicios que intervienen dentro del proceso de desarrollo de la educación virtual.

Las plataformas $\mathrm{MOOC}$ siguen evolucionando $\mathrm{y}$ para garantizar su permanencia deben ampliar sus servicios y productos, siendo factores intangibles ya que varían constantemente de acuerdo a las necesidades de los clientes, y que le dan cierta complejidad tecnológica, por lo que se hace interesante el estudio de estas plataformas prestadoras de educación no presencial. 


\section{REFERENCIAS BIBLIOGRÁFICAS}

Albornoz, M. (1994). Indicadores en ciencia y tecnología. Centro de Eargestudios e Investigaciones. Universidad Nacional de Quilmes, Buenos aires - Argentina, 133-144.

Alemán de la Garza, S. T. (Febrero de 2015). Indicadores de calidad pedagágica para el diseño de un curso en línea masivo y abierto de actualización docente. RUSC. Universities and Knowledge, págs. 104119.

Astros, I. J. (14 de Septiembre de 2015). monografias.com. Obtenido de Indicadores de gestión y medición del desempeño: https://www.monografias. com/usuario/perfiles/iva_n_turmero_ astros

Bailador, M. E. (2014). Análisis y pautas para el desarrollo de MOOCs: Estudio de caso en UNED COMA: Alemán para hispanohablantes: Nociones fundamentales. FACULTAD DE EDUCACIÓN Trabajo fin de Magister, UNIVERSIDAD NACIONAL DE EDUCACIÓN A DISTANCIA.

Bartolomè, A. (2015). ¿Son los MOOC una alternativa de aprendizaje? Comunicar, No. 44.

Castells, M. (1997). La era de la información: economía, sociedad y cultura. Madrid.

Collazos, A. (2014). Revista Educaciòn Virtual. ¿Qué tanta acogida tiene un MOOC en Coursera?

DNP, D. N. (2009). Guía metodologica para la formulacion de indicadores. Obtenido de https://colaboracion.dnp.gov.co/ CDT/Inversiones $\% 20 y \% 20$ finanzas $\% 20$ pblicas/Guia $\% 20$ Metodologica $\% 20$
Formulacion\%20-\%202010.pdf

DNP, D. N. (2013). Guía metodologica para el seguimiento a la gestion del DNP. Obtenido de https://colaboracion.dnp. gov.co/CDT/DNP/RD-G01-Guia\%20 metodologica $\% 20$ para $\% 20$ el $\% 20$ seguimiento\%20al\%20SGC.Pu.pdf

Eloy, L. M. (2015). Análisis e implicaciones del impacto del. Comunicar, págs. 73-80.

Escribano, E. A. (2014). Clasificación de los medios de evaluación en los MOOC. Revista Electrónica de Tecnología Educativa (EDUTEC).

Garrido, M. F. (2003). Formación basada en las Tecnologías de la Información y Comunicación: Análisis didáctico del proceso de enseñanza-aprendizaje. Tarragona.

Hernández, 1. A. (2015). Análisis y diseño de procesos, recursos y prácticas formativas .Estudio de las posibilidades educativas de los MOOC. Universidad de Salamanca: https://gredos.usal.es/jspui/ bitstream/10366/125846/1/2015_TFM_ Hern\%C3\%A1ndezMuriel\%2C\%20 \%C3\%81Ivaro_Estudio $\% 20 \mathrm{de} \% 20$ I a s $\% 20$ pr $\%$ C $3 \%$ A 1 cticas $\% 20$ educativas $\% 20 \mathrm{de} \% 20 \mathrm{los} \% 20 \mathrm{MOOC}$. pdf.

Kinash, S. (04 de Diciembre de 2013). MOOCing About MOOCs. (Technology) Recuperado el 01 de Octubre de 2017, de https://www. educationtechnologysolutions.com. au/2013/12/moocing-about-moocs/

Lancho, M. (2014). MOOC's and SPOC's (Small Private Online Courses): possibilities for teacher education. Revista semestral de divulgación científica, págs. 6-17. 
Laurillard, D. (2012). Building Pedagogical Patterns for Learning and Tecnology. New York: Routledge.

León, Y. R. (21 de 01 de 2014). Sistemas gestores de contenidos: una mirada desde. Revista Cubana de ACIMED, págs. 3-17.

Lorente, C. F. (26 de septiembre de 2017). Blogthinkbig.com. Obtenido de Conoce a nuestros autores : https://blogthinkbig. com/15-plataformas-de-cursos-moocpara-aprender-por-tu-cuenta

Meiss, P. (2017). Ventajas y desventajas de los cursos MOOC. Obtenido de Emagister: https://www.emagister.com/blog/cursosmooc-ventajas-y-desventajas/

MINTIC. (s.f.). Arquitectura TI Colombia marco de referencia Gobierno Indicadores. Obtenido de http://www.mintic.gov. co/arquitecturati/630/articles-8830_ indicadores.pdf.

Olmo, L. (07 de Julio de 2017). TIBbeat. Obtenido de 30 plataformas de todo el mundo con cursos MOOC gratuitos: https://www. ticbeat.com/educacion/30-plataformasde-todo-el-mundo-con-cursos-moocgratuitos/

Peré, N. (2017). APUNTES PARA ANALIZAR LA RELACIÓN ENTRE INNOVACIÓN, TIC Y FORMACIÓN PEDAGÓGICO-DIDÁCTICA. En Praxis \& Saber (págs. 15-33). http:// dx.doi.org/10.19053/22160159. v7.n15.2016.5721.

Pilkey, B. (2015). MOOCs, e-learning and beyond: exploring the future of virtual built environment teaching. the University College London.
Ruiz, C. (octubre de 2015). The MOOC: ¿An alternative model for higher education? Revista de innovaciòn educativa, pág. Vol. 7 Núm. 2.

S. T. Alemàn de la Garza, G. Z. (2015). Indicadores de calidad pedagògica para el diseño de un curso en lìnea masivo y abierto de actualizaciòn docente. RUSC. Universities and Knowledge, 104-119.

Viloria, A. (2019). Edu Trends.

Zapata, A. M. (19 de octubre de 2017). La tecnologia. Obtenido de Tecnologia de equipo, operacion y producto: http:// latecnologia1b.blogspot.com/2017/10/ tecnologia-de-equipo.html 